\title{
From Subtle to Substantial: Role of Metal Ions on $\pi-\pi$ Interactions ${ }^{\dagger}$
}

\author{
A. Srinivas Reddy, Dolly Vijay, G. Madhavi Sastry and G. Narahari Sastry* \\ Molecular Modeling Group, Organic Chemical Sciences, Indian Institute of Chemical \\ Technology, Tarnaka, Hyderabad 500 007, India \\ gnsastry@yahoo.com
}

\section{Supporting Information}

(22 pages including this page)

Table S1: Statistics of various motifs present in PDB and CSD. All the proteins containing the

five chosen metals $\left(\mathrm{m}=\mathrm{Li}^{+}, \mathrm{Na}^{+}, \mathrm{K}^{+}, \mathrm{Mg}^{2+}\right.$, and $\left.\mathrm{Ca}^{2+}\right)$ were taken. The number of motifs with either M- $\pi$ or M- $\pi-\pi$ were taken for analysis (column 3). These motifs were further divided into $\mathrm{M}-\pi, \mathrm{M}-\pi-\pi$, and SW types. For comparison the total number of metal ions and $\pi-\pi$ motifs were also given. In CSD only the number of structures were considered. All the structures with $\mathrm{M}-\pi-\pi$ were also included in the structures with $\mathrm{M}-\pi$.

Table S2: PDB reference codes for all the structures exhibiting interactions

Table S3: CSD reference codes for all the structures exhibiting interactions

Table S4: $\pi-\pi$ interaction energies (in $\mathrm{kcal} / \mathrm{mol}$ ) of the various benzene dimers in the presence of metal ions at MP2/6-31G* level of theory.

Table S5: Interaction energies of metal ions with the benzene dimers at MP2/6-31G* level of theory.

Table S6: Geometries (in $\AA$ ) of the various metal- $\pi-\pi$ complexes of benzene dimers obtained at the MP2/6-31G* level of theory.

Table S7: The metal ion affinities (in $\mathrm{kcal} / \mathrm{mol}$ ) of benzene monomer in the presence and absence of another benzene at the MP2/6-31G* and MP2/6-311++G** level of theory.

Table S8: Total energies (in hartrees) of $\mathrm{M}-\pi-\pi$ complexes at MP2/6-31G* level of theory. 
Table S9: Total energies (in hartrees) of $\mathrm{M}-\pi-\pi$ complexes at MP2/6-311++G** level of theory.

Scheme S1. The two cation- $\pi$ configurations considered in the study along with theirMP2/6311++G** interaction energies (in $\mathrm{kcal} / \mathrm{mol}$ ). The metal ion approach is along the $\Pi$ face in BM1 while in BM2 is along the molecular plane.

Figure S1: Statistics on the PDB with varying cutoff distances between the centroids of the aromatic rings and the metal ions.

Figure S2: Statistics of $\mathrm{M}-\pi$ and $\mathrm{M}-\pi-\pi$ motifs in PDB considering the imidazole ring of the histidine moiety as the second $\pi$ but located at a distance larger than the cutoff value of $7 \AA$ (a) and $6 \AA$ (b)

Figure S3: Mulliken charges of the carbon atoms in various cation $-\pi-\pi$ conformations considered. In all the complexes the carbon atoms C1-C6 correspond to the benzene ring far from the metal ion, where as the $\mathrm{C} 7-\mathrm{C} 12$ correspond to the nearer benzene ring to the metal ion.

Figure S4: Total atomic charges of the carbon atoms in various cation $-\pi-\pi$ conformations considered. In all the complexes the carbon atoms C1-C6 correspond to the benzene ring far from the metal ion, where as the $\mathrm{C} 7-\mathrm{C} 12$ correspond to the nearer benzene ring to the metal ion. 
Table S1. Statistics of number of motifs and structures containing the motifs present in PDB and CSD respectively. All the proteins containing the five chosen metals $\left(\mathrm{m}=\mathrm{Li}^{+}, \mathrm{Na}^{+}, \mathrm{K}^{+}\right.$, $\mathrm{Mg}^{2+}$, and $\mathrm{Ca}^{2+}$ ) were taken. The number of motifs with either $\mathrm{M}-\pi$ or $\mathrm{M}-\pi-\pi$ were taken for analysis (column 3). These motifs were further divided into $M-\pi, M-\pi-\pi$, and SW types. For comparison the total number of metal ions and $\pi-\pi$ motifs are also given. In CSD only the number of structures were considered wherein structures with $M-\pi-\pi$ are also included in the structures with $\mathrm{M}-\pi$.

\begin{tabular}{ccccccccccccc}
\hline & $\begin{array}{c}\text { No. of } \\
\text { proteins }\end{array}$ & $\begin{array}{c}\text { No. of } \\
\text { proteins } \\
\text { having } \\
\text { motifs }\end{array}$ & $\begin{array}{c}\text { \% of } \\
\text { proteins } \\
\text { having } \\
\text { motifs }\end{array}$ & $\begin{array}{c}\text { No. of } \\
\text { in data set }\end{array}$ & $\begin{array}{c}\text { No. of } \\
\text { only } \\
\text { M- } \pi \\
\text { motifs }\end{array}$ & $\begin{array}{c}\text { No. of } \\
\text { M- } \pi-\pi \\
\text { motifs }\end{array}$ & $\begin{array}{c}* \text { Total } \\
\pi-\pi \\
\text { motifs }\end{array}$ & $\begin{array}{c}\text { \% of } \\
\text { M- } \pi-\pi \\
\text { motifs } \\
\text { of total } \\
\pi-\pi\end{array}$ & $\begin{array}{c}\text { No. of } \\
\text { SW } \\
\text { motifs } \\
\text { (PDB) }\end{array}$ & $\begin{array}{c}\text { No. of } \\
\text { structures } \\
\text { having } \\
\text { M- } \pi \\
\text { motifs }\end{array}$ & $\begin{array}{c}\text { No. of } \\
\text { structures } \\
\text { having } \\
\text { M- } \pi-\pi \\
\text { motifs }\end{array}$ \\
$\mathrm{Li}^{+}$ & 9 & 7 & 77.8 & 14 & 3 & 12 & 156 & 7.69 & 6 & 1612 & 1355 \\
$\mathrm{Na}^{+}$ & 310 & 153 & 49.0 & 734 & 98 & 726 & 14599 & 4.97 & 555 & 1071 & 988 \\
$\mathrm{~K}^{+}$ & 131 & 68 & 55.0 & 516 & 104 & 219 & 7730 & 2.83 & 84 & 900 & 828 \\
$\mathrm{Mg}^{2+}$ & 720 & 328 & 45.8 & 1793 & 313 & 1689 & 34463 & 4.90 & 635 & 364 & 329 \\
$\mathrm{Ca}^{2+}$ & 690 & 396 & 57.4 & 2018 & 337 & 1378 & 40374 & 3.41 & 1043 & 178 & 168 \\
\hline
\end{tabular}

- With plane-plane angle $>20^{\circ}$ 
Table S2: PDB reference codes for all the structures exhibiting interactions

\begin{tabular}{|c|c|c|c|c|c|c|c|c|}
\hline Li & $1 \mathrm{~J} 9 \mathrm{M}$ & $1 \mathrm{SFH}$ & $2 \mathrm{BOK}$ & $1 \mathrm{G} 8 \mathrm{M}$ & $1 \mathrm{DOA}$ & 1IR3 & $1 \mathrm{~N} 8 \mathrm{I}$ & $1 \mathrm{RBL}$ \\
\hline 1DGD & 1JG2 & $1 \mathrm{SOO}$ & 2GEP & 1GJV & 1DXE & 1IRU & 1NE9 & 1RG9 \\
\hline $1 \mathrm{E} 5 \mathrm{~K}$ & $1 \mathrm{JJU}$ & $1 \mathrm{~T} 64$ & 2HLP & $1 \mathrm{GKZ}$ & $1 \mathrm{E} 0 \mathrm{~J}$ & 1IV3 & $1 \mathrm{NF} 3$ & 1RIF \\
\hline $1 \mathrm{MGW}$ & $1 \mathrm{JMM}$ & $1 \mathrm{~T} 6 \mathrm{~B}$ & 2IZC & 1GUP & 1E22 & 1IWE & 1NJ1 & 1RMW \\
\hline $1 \mathrm{NQJ}$ & $1 \mathrm{JMO}$ & $1 \mathrm{~T} 76$ & 3FCT & 1GUQ & 1E3D & 1IWL & 1NLK & 1RRP \\
\hline $1 \mathrm{RKB}$ & $1 \mathrm{JMS}$ & 1TC8 & & $1 \mathrm{H} 54$ & 1E96 & 1IZC & 1NMP & $1 \mathrm{RVK}$ \\
\hline 1TW6 & 1JTP & 1TG7 & $\mathbf{K}$ & 1IH7 & 1E9I & $1 \mathrm{~J} 34$ & $1 \mathrm{NQE}$ & 1RYA \\
\hline \multirow[t]{2}{*}{ 2ONE } & $1 \mathrm{JZZ}$ & 1THM & \multirow{2}{*}{$1 \mathrm{~A} 5 \mathrm{~A}$} & $1 \mathrm{IJV}$ & $1 \mathrm{EC} 7$ & $1 \mathrm{~J} 3 \mathrm{~W}$ & 1NR9 & 1RYP \\
\hline & $1 \mathrm{JZN}$ & 1TRZ & & 1IWB & $1 \mathrm{ECB}$ & $1 \mathrm{~J} 58$ & 1NUE & $1 \mathrm{~S} 1 \mathrm{C}$ \\
\hline $\mathbf{N a}$ & $1 \mathrm{~K} 2 \mathrm{X}$ & $1 \mathrm{TXZ}$ & \multirow{2}{*}{$\begin{array}{l}1 \mathrm{~A} 9 \mathrm{X} \\
1 \mathrm{AKD}\end{array}$} & 1IWP & $1 \mathrm{EFC}$ & $1 \mathrm{~J} 7 \mathrm{~L}$ & $1 \mathrm{NVU}$ & $1 \mathrm{~S} 1 \mathrm{M}$ \\
\hline $1 \mathrm{~A} 5 \mathrm{U}$ & $1 \mathrm{KA} 0$ & 1U02 & & $1 \mathrm{~J} 51$ & $1 \mathrm{EQR}$ & $1 \mathrm{JBW}$ & 1NY5 & 1S4E \\
\hline $1 \mathrm{~A} 7 \mathrm{~T}$ & $1 \mathrm{KJQ}$ & $1 \mathrm{U} 4 \mathrm{~J}$ & $1 \mathrm{AOP}$ & $1 \mathrm{JBS}$ & $1 \mathrm{ESN}$ & 1JM1 & $1 \mathrm{NYK}$ & 1S5J \\
\hline 1AG9 & $1 \mathrm{KJT}$ & 1UD2 & $1 \mathrm{AX} 4$ & $1 \mathrm{JBT}$ & 1EYE & 1JM6 & 1NZI & 1S83 \\
\hline $1 \mathrm{AVF}$ & $1 \mathrm{~L} 8 \mathrm{~S}$ & 1UR5 & $1 \mathrm{BEU}$ & $1 \mathrm{JDB}$ & 1F0J & 1JMS & 1004 & 1SAW \\
\hline 1B57 & 1L9B & 1UUO & $1 \mathrm{BO} 8$ & 1JF8 & $1 \mathrm{~F} 3 \mathrm{~F}$ & $1 \mathrm{JP} 4$ & $101 \mathrm{~T}$ & 1SGJ \\
\hline $1 \mathrm{BCH}$ & 1M5Q & 1UYP & $1 \mathrm{BP} 0$ & $1 \mathrm{JFV}$ & 1F51 & $1 \mathrm{JZZ}$ & 1O5Q & $1 \mathrm{SH} 3$ \\
\hline 1BG4 & 1M65 & 1V1R & $1 \mathrm{BP} 6$ & 1JSC & $1 \mathrm{~F} 5 \mathrm{~N}$ & 1K4I & $106 \mathrm{Y}$ & 1SHK \\
\hline 1BUN & 1M9M & 1V47 & 1BPJ & $1 \mathrm{JVJ}$ & 1F6B & $1 \mathrm{~K} 5 \mathrm{D}$ & 1OBD & $1 \mathrm{SHQ}$ \\
\hline $1 \mathrm{C} 1 \mathrm{D}$ & 1MG2 & 1V54 & 1BXR & $1 J W V$ & $1 \mathrm{~F} 8 \mathrm{M}$ & $1 \mathrm{~K} 77$ & 1OBW & 1SIX \\
\hline $1 \mathrm{C} 82$ & 1MWV & 1V6P & 1C1D & $1 \mathrm{~K} 28$ & 1F97 & 1KA1 & $10 C 7$ & $1 \mathrm{SJC}$ \\
\hline 1CFB & $1 \mathrm{~N} 82$ & $1 \mathrm{~V} 70$ & $1 \mathrm{C} 1 \mathrm{X}$ & $1 \mathrm{KAX}$ & 1F9Y & $1 \mathrm{KA} 21 \mathrm{~K}$ & 1OD5 & $1 \mathrm{SO} 2$ \\
\hline $1 \mathrm{CQX}$ & 1NA0 & $1 \mathrm{~V} 8 \mathrm{Z}$ & $1 \mathrm{C} 30$ & $1 \mathrm{KAY}$ & 1FRF & $\mathrm{AO}$ & 1OL5 & $1 \mathrm{SOF}$ \\
\hline 1D3Y & 1NSZ & 1VBW & $1 \mathrm{C} 3 \mathrm{O}$ & 1KAZ & 1FVU & $1 \mathrm{KCZ}$ & 1ONE & 1SU2 \\
\hline 1DPY & 1NXD & 1VI6 & $1 \mathrm{C} 7 \mathrm{~J}$ & & 1FXX & $1 \mathrm{KEK}$ & 10T8 & 1SXI \\
\hline 1DX5 & 1NZA & 1VK1 & 1CE8 & Mg & $1 \mathrm{G} 29$ & $1 \mathrm{KH} 3$ & 1OU8 & $1 \mathrm{~T} 1 \mathrm{~S}$ \\
\hline $1 \mathrm{E} 43$ & $101 Z$ & 1VMJ & $1 \mathrm{CPE}$ & $1 \mathrm{~A} 49$ & $1 \mathrm{G} 87$ & $1 \mathrm{KJQ}$ & $10 U O$ & $1 \mathrm{~T} 3 \mathrm{~T}$ \\
\hline 1EJ2 & $104 Z$ & 1VR5 & $1 \mathrm{CPG}$ & $1 \mathrm{AIH}$ & $1 \mathrm{G} 8 \mathrm{~F}$ & $1 \mathrm{KJY}$ & $1 \mathrm{OY0}$ & $1 \mathrm{~T} 57$ \\
\hline 1EMS & 1068 & 1VZQ & $1 \mathrm{CS} 0$ & $1 \mathrm{AMU}$ & $1 \mathrm{G} 9 \mathrm{G}$ & $1 \mathrm{KKR}$ & $1 \mathrm{OZH}$ & 1T8Q \\
\hline $1 \mathrm{EXC}$ & 1OAN & 1W15 & 1D7R & $1 \mathrm{AO} 0$ & 1GGQ & $1 \mathrm{KMI}$ & 1P31 & $1 \mathrm{~T} 9 \mathrm{~B}$ \\
\hline 1F6D & IOAR & 1W37 & 1D7S & $1 \mathrm{AOX}$ & 1GK8 & $1 \mathrm{KMQ}$ & 1P4D & $1 \mathrm{TAZ}$ \\
\hline $1 \mathrm{G} 1 \mathrm{~S}$ & 1OB0 & 1W9S & 1D7U & 1AR1 & $1 \mathrm{GKB}$ & $1 \mathrm{KQP}$ & 1P9B & 1TBF \\
\hline $1 \mathrm{G} 29$ & 10D8 & 1W9X & $1 \mathrm{D} 7 \mathrm{~V}$ & 1AUK & $1 \mathrm{GKZ}$ & $1 \mathrm{KTG}$ & 1PA1 & $1 \mathrm{TBM}$ \\
\hline $1 \mathrm{G} 5 \mathrm{H}$ & 1ODZ & 1WKY & $1 \mathrm{DHP}$ & $1 \mathrm{~B} 6 \mathrm{~S}$ & 1GMI & $1 \mathrm{KVK}$ & 1PBJ & 1TE6 \\
\hline $1 \mathrm{G} 60$ & $1 \mathrm{OIH}$ & 1WNW & $1 \mathrm{DIO}$ & $1 \mathrm{~B} 8 \mathrm{C}$ & 1GQI & $1 \mathrm{KXG}$ & 1PFK & $1 \mathrm{TFR}$ \\
\hline 1G8I & 1P9E & 1WPC & 1DK1 & 1BK4 & 1GS6 & $1 \mathrm{LOO}$ & $1 \mathrm{POX}$ & 1TH8 \\
\hline $1 \mathrm{GEN}$ & 1PJ5 & 1XAR & 1DKA & 1BL3 & $1 \mathrm{H} 1 \mathrm{~L}$ & $1 \mathrm{~L} 3 \mathrm{R}$ & 1PVD & $1 \mathrm{TJO}$ \\
\hline $1 \mathrm{GLH}$ & 1POX & $\begin{array}{l}1 \mathrm{XJ} 4 \\
1 \mathrm{YV}\end{array}$ & 1DOI & 1BWV & $1 \mathrm{H} 3 \mathrm{I}$ & $1 \mathrm{~L} 5 \mathrm{Y}$ & 1PYM & $1 \mathrm{TQY}$ \\
\hline 1GMM & $\begin{array}{l}\text { IQ6X } \\
\text { IDen }\end{array}$ & 1XK8 & 1DZ4 & $1 \mathrm{BXZ}$ & 1H56 & 1LDF & 1Q3B & 1TT4 \\
\hline 1GN0 & 1Q8C & 1XSS & 1DZ6 & 1BZY & $1 \mathrm{HBN}$ & 1LM3 & 1Q5H & 1TW1 \\
\hline $1 \mathrm{GVH}$ & 1Q9I & 1XXF & 1DZ8 & 1C1Q & 1HE1 & 1LNZ & 1Q6O & 1TWY \\
\hline $1 \mathrm{H} 16$ & 1QOP & 1Y0B & 1DZ9 & $1 \mathrm{C} 1 \mathrm{Y}$ & 1HE8 & $1 \mathrm{M} 15$ & 1Q92 & $1 \mathrm{TXU}$ \\
\hline $1 \mathrm{H} 80$ & 1QY1 & 1YCE & 1EEX & $1 \mathrm{CHN}$ & $1 \mathrm{HFV} 1 \mathrm{H}$ & $1 \mathrm{M} 3 \mathrm{U}$ & 1Q9R & $1 \mathrm{TZF}$ \\
\hline $1 \mathrm{HBN}$ & 1R4P & 1YGG & $1 \mathrm{EGM}$ & $1 \mathrm{CIB}$ & I8 & 1M56 & $1 \mathrm{Q} 9 \mathrm{~W}$ & $1 \mathrm{TZZ}$ \\
\hline 1HN0 & 1R89 & 1YX1 & $1 \mathrm{EGV}$ & $1 \mathrm{CJB}$ & $1 \mathrm{HTW}$ & $1 \mathrm{M} 7 \mathrm{~B}$ & 1QB7 & $1 \mathrm{U} 3 \mathrm{D}$ \\
\hline $1 \mathrm{HNF}$ & $\begin{array}{l}\text { 1RJO } \\
\text { 1PRK }\end{array}$ & 1YY6 & $1 \mathrm{EHY}$ & $1 \mathrm{CMC}$ & 1HW6 & 1MDL & 1QBK & $1 \mathrm{U} 3 \mathrm{G}$ \\
\hline 1HTW & 1RRK & $1 \mathrm{Z2U}$ & 1ELQ & $1 \mathrm{CXZ}$ & $1 \mathrm{HYO}$ & 1MFR & 1QGQ & 1U7P \\
\hline 1HVX & 1RTQ & 1ZA2 & 1ELU & 1D3Y & 1HZ1 & 1MIW & 1QHA & 1U8Z \\
\hline $1 \mathrm{HX} 6$ & 1RVG & 1ZDN & 1EU3 & 1D5A & $1 \mathrm{I} 58$ & $1 \mathrm{MJN}$ & 1QK5 & 1UBK \\
\hline $1 \mathrm{I} 2 \mathrm{~S}$ & 1RWH & 1ZKP & $1 \mathrm{~F} 7 \mathrm{Y}$ & 1D5C & 1IAH & 1MQ4 & 1QL0 & $1 \mathrm{UKV}$ \\
\hline $1 \mathrm{I} 40$ & 1S5D & 2BEM & 1FL1 & 1DAW & 1IBR & $1 \mathrm{MUW}$ & 1QLG & 1UL1 \\
\hline 1IN0 & $\begin{array}{l}1 \mathrm{~S} 5 \mathrm{~N} \\
1 \mathrm{~S} 6 \mathrm{C}\end{array}$ & 2BGD & 1FP7 & 1DEK & $1 \mathrm{ICC}$ & $1 \mathrm{MXG}$ & 1QM4 & $1 \mathrm{UMG}$ \\
\hline $1 \mathrm{IUE}$ & $\begin{array}{l}\text { IS6G } \\
1 \mathrm{SAZ}\end{array}$ & $\begin{array}{l}\text { 2BL2 } \\
\text { 2BLE }\end{array}$ & $1 \mathrm{FT} 7$ & 1DJ9 & 1INP & 1N1B & 1QR0 & 1UPB \\
\hline & 1SAE & ZDLL & & & 1IQC & 1N52 & 1R9Z & 1VOD \\
\hline
\end{tabular}




\begin{tabular}{|c|c|c|c|c|c|c|c|c|}
\hline $1 \mathrm{~V} 25$ & 1YYQ & $1 \mathrm{C} 1 \mathrm{Y}$ & $1 \mathrm{G} 72$ & $1 \mathrm{IXX}$ & 1M9I & 1PWB & 1UEA & $1 \mathrm{Y} 10$ \\
\hline 1V54 & $1 Y Z Q$ & 1C7I & $1 \mathrm{G} 7 \mathrm{Y}$ & $1 \mathrm{~J} 1 \mathrm{~T}$ & 1MDW & 1Q39 & 1UET & $1 \mathrm{Y} 4 \mathrm{~J}$ \\
\hline $1 \mathrm{~V} 5 \mathrm{~F}$ & $1 \mathrm{Z} 08$ & $1 C 8 D$ & 1G87 & $1 \mathrm{~J} 34$ & $1 \mathrm{MIO}$ & 1Q3A & 1UF3 & 1Y7B \\
\hline 1V98 & $1 \mathrm{Z1L}$ & $1 \mathrm{C} 8 \mathrm{~N}$ & $1 \mathrm{G} 8 \mathrm{~W}$ & $1 \mathrm{~J} 4 \mathrm{G}$ & $1 \mathrm{MMQ}$ & 1Q6Z & 1UIS & 1Y93 \\
\hline 1VA6 & $1 \mathrm{Z} 2 \mathrm{C}$ & 1CB8 & 1G94 & $1 \mathrm{~J} 83$ & $1 \mathrm{MNZ}$ & 1Q7B & $1 \mathrm{UKG}$ & 1Y9I \\
\hline 1VC9 & $1 \mathrm{Z72}$ & $1 \mathrm{CDL}$ & $1 \mathrm{G} 9 \mathrm{~K}$ & $1 \mathrm{~J} 8 \mathrm{E}$ & $1 \mathrm{MPX}$ & $1 \mathrm{Q} 8 \mathrm{~F}$ & 1UL3 & $1 Y D N$ \\
\hline 1VG8 & $1 \mathrm{ZBD}$ & 1CGE & $1 \mathrm{GBG}$ & $1 \mathrm{~J} 9 \mathrm{~L}$ & 1MU5 & 1QHD & $1 \mathrm{ULV}$ & 1YDY \\
\hline $1 \mathrm{VKP}$ & $1 \mathrm{ZC} 3$ & 1CGT & $1 \mathrm{GCA}$ & $1 \mathrm{JAE}$ & $1 \mathrm{MVE}$ & $1 \mathrm{QHO}$ & $1 \mathrm{UMM}$ & $1 Y F Q$ \\
\hline 1VL8 & 1ZE1 & $1 \mathrm{CIU}$ & $1 \mathrm{GCY}$ & 1JC9 & $1 \mathrm{MVQ}$ & 1QIB & $1 \mathrm{UMN}$ & 1YTZ \\
\hline 1VR0 & 1ZED & 1CLC & $1 \mathrm{GGZ}$ & 1JI1 & $1 \mathrm{MXE}$ & 1QLA & $1 \mathrm{UMV}$ & 1YWT \\
\hline 1VRG & $1 \mathrm{ZK} 4$ & $1 \mathrm{CLV}$ & 1GK9 & 1JIA & $1 \mathrm{MXG}$ & 1QLE & 1UOW & $1 Y X H$ \\
\hline 1VRP & $1 \mathrm{ZKE}$ & 1CM4 & $1 \mathrm{GKD}$ & $1 \mathrm{JIW}$ & 1N28 & 1QMD & 1UP8 & $1 \mathrm{Z} 32$ \\
\hline 1VRT & $1 \mathrm{ZKL}$ & $1 \mathrm{CPN}$ & 1GP7 & $1 \mathrm{JMJ}$ & $1 \mathrm{~N} 2 \mathrm{~K}$ & 1QNI & 1UPS & $1 \mathrm{Z} 6 \mathrm{O}$ \\
\hline 1VSD & 1ZPD & 1CRU & 1GTT & $1 \mathrm{JRO}$ & 1N47 & $1 \mathrm{QNW}$ & 1UR4 & $1 \mathrm{Z70}$ \\
\hline $1 \mathrm{~W} 0 \mathrm{H}$ & 1ZS9 & $1 \mathrm{CVR}$ & 1GU6 & 1JTD & $1 \mathrm{~N} 9 \mathrm{E}$ & 1R0R & 1US1 & 1ZED \\
\hline $1 \mathrm{~W} 0 \mathrm{~N}$ & 1ZY5 & $1 \mathrm{CXL}$ & $1 \mathrm{GUI}$ & 1JX6 & $1 \mathrm{NBC}$ & 1R17 & 1UX6 & 1ZIV \\
\hline $1 \mathrm{~W} 23$ & 2BEK & $1 \mathrm{CYG}$ & $1 \mathrm{GWU}$ & $1 \mathrm{JZN}$ & 1NEK & 1R1Z & 1UXX & 1ZR0 \\
\hline 1W2Y & 2BFR & 1D2S & $1 \mathrm{GXO}$ & 1K1X & 1NG0 & 1R64 & 1UYY & 2AAA \\
\hline 1W78 & 2BJI & 1DAN & $1 \mathrm{GXR}$ & $1 \mathrm{~K} 3 \mathrm{I}$ & 1NIW & $1 \mathrm{RDO}$ & 1UZJ & $2 \mathrm{AYH}$ \\
\hline 1W79 & $2 \mathrm{BKU}$ & $1 \mathrm{DBN}$ & $1 \mathrm{HOH}$ & $1 \mathrm{~K} 6 \mathrm{~S}$ & 1NKQ & 1RHI & 1V00 & 2BF0 \\
\hline 1WA5 & $2 \mathrm{BOV}$ & 1DE4 & $1 \mathrm{H} 1 \mathrm{~A}$ & $1 \mathrm{~K} 7 \mathrm{I}$ & $1 \mathrm{NL0}$ & 1RM8 & 1V04 & 2BIB \\
\hline 1WC1 & $2 \mathrm{BVC}$ & 1DHK & $1 \mathrm{H} 30$ & $1 \mathrm{~K} 90$ & 1NL1 & 1RQ5 & $1 \mathrm{~V} 0 \mathrm{~A}$ & $2 \mathrm{BKH}$ \\
\hline 1WDC & 2NGR & 1DJX & $1 \mathrm{H} 3 \mathrm{G}$ & $1 \mathrm{~K} 94$ & 1NLS & 1RRO & $1 \mathrm{~V} 1 \mathrm{G}$ & $2 \mathrm{LTN}$ \\
\hline $1 \mathrm{WDD}$ & 2RUS & 1DL2 & 1H6G & $1 \mathrm{~K} 9 \mathrm{~J}$ & $1 \mathrm{NPC}$ & 1RU4 & $1 \mathrm{~V} 3 \mathrm{~W}$ & 2MAS \\
\hline $1 \mathrm{WPV}$ & $2 \mathrm{TCT}$ & 1DSY & 1H6L & $1 \mathrm{~K} 9 \mathrm{U}$ & 1NPS & 1RWY & 1V6I & $2 \mathrm{MCM}$ \\
\hline $1 \mathrm{WPW}$ & 2TPS & 1DTL & $1 \mathrm{H} 80$ & 1KA1 & 1NQD & $1 \mathrm{RXO}$ & 1V73 & 2MSB \\
\hline 1WUF & $2 \mathrm{UAG}$ & 1DV8 & $1 \mathrm{H} 93$ & $1 \mathrm{KAP}$ & 1NT0 & 1RZ5 & $1 \mathrm{~V} 7 \mathrm{~W}$ & 2POR \\
\hline 1WXI & 2XIM & $1 \mathrm{DX} 5$ & 1H9W & $1 \mathrm{~KB} 0$ & 1NW1 & $1 \mathrm{SOE}$ & 1V97 & 2PVB \\
\hline $1 \mathrm{WYW}$ & 3FCT & $1 \mathrm{DYO}$ & $1 \mathrm{HCU}$ & 1KEK & $1 \mathrm{NXC}$ & $1 \mathrm{~S} 3 \mathrm{X}$ & $1 \mathrm{VCL}$ & $3 \mathrm{CSU}$ \\
\hline $1 \mathrm{WYX}$ & 4PAL & $1 \mathrm{E} 43$ & $1 \mathrm{HDF}$ & $1 \mathrm{KFQ}$ & 1NZI & 1S6B & 1VEM & 3DNI \\
\hline 1X1R & 4PFK & $1 \mathrm{E} 8 \mathrm{~A}$ & $1 \mathrm{HDH}$ & $1 \mathrm{KKT}$ & $104 Y$ & 1S6C & $1 \mathrm{VLF}$ & $3 \mathrm{FIB}$ \\
\hline 1X8B & 4RUB & 1EA7 & $1 \mathrm{HFC}$ & $1 \mathrm{KLI}$ & $106 \mathrm{~S}$ & 1SAT & 1VRK & 4CPV \\
\hline 1XD3 & 4XIA & 1EB7 & 1HJ9 & 1KP4 & $107 \mathrm{~L}$ & $1 \mathrm{SBF}$ & 1VSI & $4 \mathrm{SBV}$ \\
\hline $1 \mathrm{XDN}$ & & 1EJ8 & 1HL5 & $1 \mathrm{KSC}$ & $109 \mathrm{I}$ & 1SGX & 1W07 & 4SGB \\
\hline 1XDP & $\mathbf{C a}$ & 1EL1 & $1 \mathrm{HQL}$ & 1KV9 & $1 \mathrm{OAC}$ & 1SL4 & $1 \mathrm{~W} 0 \mathrm{~N}$ & $5 \mathrm{CHY}$ \\
\hline 1XED & $1 \mathrm{~A} 25$ & 1EN7 & $1 \mathrm{HQV}$ & $1 \mathrm{KXQ}$ & $1 \mathrm{OAH}$ & $1 \mathrm{SLU}$ & $1 \mathrm{~W} 0 \mathrm{P}$ & 5PAL \\
\hline 1XFI & $1 \mathrm{~A} 75$ & $1 \mathrm{~F} 4 \mathrm{~N}$ & 1HT6 & $1 \mathrm{KXT}$ & 1OB0 & 1SMP & 1W15 & 7TAA \\
\hline 1XHX & $1 \mathrm{~A} 8 \mathrm{~B}$ & 1F7L & 1HUP & 1L6J & 1OBR & $1 \mathrm{SMV}$ & $1 \mathrm{~W} 6 \mathrm{~S}$ & $830 \mathrm{C}$ \\
\hline $1 \mathrm{XMX}$ & 1AG9 & $1 \mathrm{~F} 9 \mathrm{~K}$ & 1HV5 & 1L6R & 1OD3 & $1 \mathrm{SNN}$ & 1W9X & 8TLN \\
\hline $1 \mathrm{XPY}$ & $1 \mathrm{AJJ}$ & 1FAK & $1 \mathrm{HVX}$ & $1 \mathrm{~L} 7 \mathrm{~L}$ & $1 \mathrm{OH} 4$ & 1SPJ & $1 \mathrm{WBF}$ & \\
\hline 1XQA & $1 \mathrm{AJK}$ & 1FE5 & $1 \mathrm{HX} 0$ & $1 \mathrm{~L} 8 \mathrm{~S}$ & $1 \mathrm{OKG}$ & 1SRA & 1WC5 & \\
\hline 1XS1 & $1 \mathrm{AJO}$ & $1 \mathrm{FKQ}$ & $1 \mathrm{HY} 7$ & $1 \mathrm{LBZ}$ & $10 L P$ & 1SU4 & 1WDA & \\
\hline 1XSS & 1AR1 & 1FLG & $1 \mathrm{HYO}$ & 1LE6 & 1OS1 & 1SUI & $1 \mathrm{WKY}$ & \\
\hline 1XTJ & 1AUI & 1FNY & $1 \mathrm{I} 0 \mathrm{~V}$ & 1LED & 1OU9 & 1SZB & 1WL7 & \\
\hline 1XTQ & 1AVA & 1FOB & $1 \mathrm{I} 40$ & 1LEN & $10 Y G$ & $1 \mathrm{~T} 9 \mathrm{H}$ & 1WMD & \\
\hline 1XX1 & $1 \mathrm{AXK}$ & 1FS7 & $1 \mathrm{II} 76$ & $1 \mathrm{LOE}$ & $10 Z 6$ & $1 \mathrm{TCO}$ & $1 \mathrm{WMZ}$ & \\
\hline $1 \mathrm{Y} 2 \mathrm{~K}$ & $1 \mathrm{~B} 9 \mathrm{O}$ & $1 \mathrm{FSU}$ & $1 \mathrm{I} 8 \mathrm{~A}$ & $1 \mathrm{LPP}$ & $1 \mathrm{P} 49$ & $1 \mathrm{TDQ}$ & $1 \mathrm{WND}$ & \\
\hline 1Y9I & $1 \mathrm{BAG}$ & 1FWX & $1 \mathrm{I} 9 \mathrm{Z}$ & $1 \mathrm{LQV}$ & 1P6O & 1TF4 & 1WPC & \\
\hline 1YD1 & 1BG3 & 1FX5 & 1IA6 & 1LRW & 1PAM & $1 \mathrm{TGM}$ & $1 \mathrm{WYG}$ & \\
\hline 1YEY & $1 \mathrm{BJJ}$ & 1FXW & 1ID5 & 1LU1 & 1PEX & 1TL9 & $1 \mathrm{X} 1 \mathrm{~J}$ & \\
\hline 1YFR & 1BK9 & $1 \mathrm{FZC}$ & 1IJL & $1 \mathrm{LVU}$ & 1PJX & 1TLQ & $1 X 7 \mathrm{I}$ & \\
\hline 1YM3 & $1 \mathrm{BQB}$ & 1FZD & $1 \mathrm{IME}$ & $1 \mathrm{LWU}$ & 1PMH & $1 \mathrm{TN} 3$ & $1 \mathrm{XCB}$ & \\
\hline 1YMQ & 1BU3 & $1 \mathrm{G} 20$ & $1 \mathrm{IOD}$ & $1 \mathrm{M} 1 \mathrm{~N}$ & 1PMZ & $1 \mathrm{TVG}$ & $1 \mathrm{XHB}$ & \\
\hline 1YVE & 1BUV & $1 \mathrm{G} 33$ & 1IQ5 & 1M56 & $1 \mathrm{POA}$ & $1 \mathrm{TXV}$ & $1 \mathrm{XO5}$ & \\
\hline 1YW0 & $1 \mathrm{BVN}$ & $1 \mathrm{G} 43$ & 1IQC & 1M63 & $1 \mathrm{POC}$ & 1U94 & $1 \mathrm{XYN}$ & \\
\hline $1 \mathrm{YXO}$ & $1 \mathrm{BYF}$ & $1 \mathrm{G} 4 \mathrm{I}$ & 1IRJ & $1 \mathrm{M} 8 \mathrm{~T}$ & 1PVA & $1 \mathrm{UCN}$ & $1 \mathrm{XZO}$ & \\
\hline
\end{tabular}


Table S3: CSD reference codes for all the structures exhibiting interactions

\begin{tabular}{|c|c|c|c|c|c|c|}
\hline Li & BENRES & CEZQED & DOFKIS & FAFZUI & GAFXIU & HAJSIU \\
\hline ABEVOS & BEQREU & CEZSOP & DOFKOY & FAGFOI & GAFXUG & HAKVEU \\
\hline ABEZOW & BERHOV & CIDZAQ & DOFQAQ & FAHBEV & GAGDUN & HAQLEQ \\
\hline ADOSAN & BESQUL & CIFDOK01 & DOJPOH & FAJKEG & GAGFAV & HAQXUS \\
\hline AGEYEQ & BEYROM01 & CIGWIY & DOJYAC & FALGAA & GAGYOC & HAYWAF \\
\hline AGICOI & BFLULI & CIGXUL & DOKGUF & FALVUJ & GAJVIW & HEPSUQ \\
\hline AGOJOV & BISVEE & CILPIW & DOKHAM & FALWAQ & GAKBAV & HEQQEZ \\
\hline AGONAL & BISZAE & CINCAD & DOMSED & FALWEU & GANBAY & HESJIY \\
\hline AGONEP & BISZUY & CIQRAV & DOQTEI & FANHIL & GASXIH & HEWCOB \\
\hline AGONIT & BIWCUG & CISPEZ & DOXDUP & FAPWUO & GATBEI & HIGQOD \\
\hline AGUHIT & BOVKOM & CISQAW & DOXFAX & FATPEV & GATQUN & HIGQUJ \\
\hline AGUHUF & BOWMUV & CIVVAE & DOXMUY & FECQUZ & GAVYUX & HILTAX \\
\hline AHAFIY & BUDJIT & CIVVIM & DOXQUC & FECRAG & GAXHIW & HIMCIP \\
\hline AJENIM & BUHHAN & CIYWUC & DOZHEF & FECWIT & GEDDOI & HISNIG01 \\
\hline AJENOS & BUJWOS & COHRAS & DUGYUZ & FEDHUR & GEDDUO & HITFEV \\
\hline AJUZEK & BUQYER & COKSEA & DUJDIV & FEJNEN & GEDWER & HITHAT \\
\hline AKACAQ & BUQYOB & COKVON & DUNYIU & FELVIB & GEJRIW & HITPAB \\
\hline AKACEU & BZLINI & COSALL & DUWYOJ & FEQGAJ & GEKFOR & HIYTEO \\
\hline AKUKIA & BZLITE & COSQIK & DUWYUP & FERVON & GEQRUP & HIZMAE \\
\hline AKUPOL & CACKOH & COZBUO & DUWZAW & FEVVOR & GEVDUG & HODGUC \\
\hline AKUQAY & CACKUN & COZJUW & DUWZEA & FEWQUT & GEVFAO & HOFYAC \\
\hline ALAMIJ & CADTUX & CROBLI & DUWZIE & FEWTAC & GEVJUM & HOGJIW \\
\hline ALOCOT & CADVIN & CUFKAP & EBUBUY & FEWWAF & GEVKAT & HOKQIH \\
\hline ALOKAN & CADVOT & CUQGAW & ECASUW & FEWWUZ & GEVKEX & HOLSOQ \\
\hline AMINEP & CAFQOP & CUQGEA & ECIHUT & FEYLEA & GEYLAX & HOLSUW \\
\hline AQINOD & CAFREH & CURWUH & EDIXUK & FEYLIE & GIBTOA & HOPTUB \\
\hline AQINUJ & CAFRIL & CUSFEB & EDIYAR & FIBQIQ & GIBWET & HOPVEN \\
\hline AQIPIZ & CAGLOM & CUSFOL & EFABES & FIBYOE & GIJWOL & HOPVIR \\
\hline AQIPOF & CAKCEX & CUWTUJ & EGAQUY & FIDWEU & GIKXAZ & HOQGEZ \\
\hline AREYOL & CALKOP & CUXXIC & EGUCUE & FIDWIY & GILHAK & HOQGOJ \\
\hline ARIYOP & CALKUV & CUXXOI & EGUDAL & FIFBEB & GILHEO & HOTFOL \\
\hline AROTAC & CALQOV & DAGKOL & EGUDEP & FIGHEI & GILHIS & HOTFUR \\
\hline ASECAC & CAMNEJ & DANKUY & EJAHUS & FIQMEX & GIMPIB & HOTGIG \\
\hline ASITUR & CAMPUB & DAZWOQ & EJAJAA & FISDIU & GIMPOH & HOTJIJ \\
\hline AWEKIW & CATZAY & DEFQOU & EKAWUI & FIVYAK & GINVEE & HOTJOP \\
\hline BACDOZ & CAWREX20 & DEKJUY & EKUPEF & FIXLIH & GITNEC & HOVNIP \\
\hline BADGOD & CAWRIB20 & DELKAG & ELUVUC & FOFBEH & GOMHIZ & HOVNOV \\
\hline BAFMEB & CAWVUR & DENGUY & ELUWAJ & FOFBIL & GOMHOF & HOWYOH \\
\hline BAFMIF & CAWWAY & DEQNAO & ELUWEN & FOGTAW & GOSNUX & HOXBOL \\
\hline BAHFUM & CAXTAW & DEVJUJ & ELUWIR & FOHSUQ & GOSPAF & HOYJAG \\
\hline BAHGAT & CAXWUT & DIBXIV & EMUYEQ & FOQPEG & GOTQIP & HOYNOY \\
\hline BAJMAB & CAXXUU & DIBYES & EMUYIU & FOQPIK & GOTQOV & HOYNUE \\
\hline BAJMEF & CAXYAB & DIFHAB & ENAWOF & FOQTEK & GOTQUB & HUDZEL \\
\hline BAMTEP & CECBUH & DIJPIV & EREZEG & FORBOD & GOYMIQ & HUGJAU \\
\hline BARCED & CEGFAV & DIKRUK & EROREI & FORZOB & GOYRUH & HUJBUJ \\
\hline BASTAR & CEKMEK & DIMHOW10 & ESAXUR & FOWDEA & GUGLID & HUMCAT \\
\hline BASVAT & CEMNOX & DIVLID & ESIQAY & FUBFIR & GUGMAW & HUPZUN \\
\hline BATZAX & CEPLAK & DIXNIH02 & ETEHAM & FUBFOX & GUGMEA & HURLUB \\
\hline BAWJOY & CEPTOG & DIYGIB & EWAFUD & FUBFUD & GUNJII & HURMAI \\
\hline BAWLEQ & CEPTUM & DNPNIS & EWIWEM & FULIAO10 & GUTLOW & HURMEM \\
\hline BAWYUT & CETWED & DOBRUH01 & EWOBOH & FUWYUR & GUVJOW & HUXBOR \\
\hline BAXJOA & CETYAB & DODGUY & EWOJIJ & FUYWIF & GUWMUG & ICUNID \\
\hline BAYQUN & CEVMUL & DOFFIN & FACKOJ & FUYWUR & GUXHUC & ICUNOJ \\
\hline BEDDAQ & CEVNAS & DOFHOW & FACKUP & GABHEW & HAJGUU & IDOQIB \\
\hline $\mathrm{AO}$ & EVYUX & DOFJEN & FADHEX & GACLUS & HAJHEF & IFAMAD \\
\hline
\end{tabular}




\begin{tabular}{|c|c|c|c|c|c|c|}
\hline IFAMOR & JISJUQ & KOCFEN & LUBQUU & MOTYOJ & NUDBIX & PAFMIS \\
\hline IFOJIW & JISLAY & KOCFUD & LUGGUP & MOXJAK & NUFLAB & PAHXEB \\
\hline IFOJOC & JITGIC & KOCXUV & LUGHAW & MOYSIC & NUFWUG & PAKDIO \\
\hline IGOFAL & JITREJ & KODYOR & LUNFOP & MOZZEG & NUFXAN & PALLIX \\
\hline IGUDUJ & JIWRIQ & KOLFOG & LUNGAC & MPSIHG10 & NUFXER & PALSUQ \\
\hline IHEXEY & JIWZIY & $\mathrm{KOPCOH}$ & LUNGIK & MUMNEN & NUHFAX & PALWII \\
\hline IHIYAZ & JIXJAB & KOZWEB & LURHIP & MUSBEH & NULRER & PAPCEO \\
\hline IJIKER & JIXPIP & KUMJUX & LUTVUR & MUSBIL & NULRIV & PATKUQ \\
\hline IKULAB & JODDAH & KUPFUW & MAJNUG & MUXLIA & NURFEL & PAUELI10 \\
\hline ILECUX & JODDEL & KUSZIH & MAJPAO & MUYWUY & NUSGAJ & PAWKIH \\
\hline ILEDEI & JOHWUY & LAFBUP & MAKHEL & MUYXAF & NUWBEM & PAWLEE \\
\hline ILEFEK & JOJXOV & LAFPIR & MAKJEN & MUYXEJ & NUYVIM & PAXNEH \\
\hline ILEWAX & JOJZUD & LAGJEI & MAQDUD & MUYXIN & NUZWEK & PAYRUC \\
\hline ILEWEB & JOKDES & LAJQIW & MAVBEQ & MXPHLI & OCEJOV & PAZSAK \\
\hline ILEWIF & JOLPIJ & LAPLOD & MAWSIM & NACTIU & OCEJUB & PAZSEO \\
\hline ILOLOK & JONWIS & LAQLEU & MAYLED & NADHEF & OCESAQ & PEBQIW \\
\hline IMEPIZ & JOQNIM & LAQLIY & MAYLON & NAKCUX & OCESUK & PECLOY \\
\hline IMEPOF & JOQVOA & LAVSIK & MAZZIW & NAMBUY & OCOWIM & PEHKUI \\
\hline IMEQAS & JOQVUG & LAVSUW & MAZZUI & NAPLIM & OCUHEZ & PEJPID \\
\hline IMODET & JORHIH & LDMSBH & MEDJIO & NAXXIT & ODIWED & PEMNOK \\
\hline INDYLI & JOSTOA & LEBSEQ & MEFROE & NAZXUH & ODOVUY & PERJEB \\
\hline INFLLI10 & JOVTUJ & LEBTOB & MEHBEG & NECHEI & ODOWAF & PESMEF \\
\hline INOBES & JUBSAA & LEDQUG & MEHBIK & NECPOA & ODOWEJ & PHENLI \\
\hline IQEMAS & JUKDAU & LEFSOE & MEQVIN & NECQER & ODOWIN & PHMGLI \\
\hline IRANAQ & JUNDEB & LEGCIJ & MEQVUZ & NEHGOW & ODUDEW & PIBGAI \\
\hline IRANIY & JUNDIF & LELRID & MEQWAG & NEHXED & OFIWAB & PICMAP \\
\hline IRATIE & JUPHAD & LEMJUI & MERBIU & NEKJAO & OFIWEF & PIDHUF \\
\hline ISIZIT & JUYSIF & LEMKAP & MEVJIG & NELCUC & OFONEC & PIHWUY \\
\hline ISOXOD & JUYSOL & LEZZAR & MEWQEK & NELDAJ & OGOPEF & PIPGOK \\
\hline IWOWUM & JUYWAB & LIANTR & MEXPUA & NELZUZ & OHAHOU & PITBUP \\
\hline JABVAK & KABWIU & LIBPNI & MEXQAH & NEMBAI & OHAHUA & PITCAW \\
\hline JABVEO & KABWOA & LIBZDM & MEXQEL & NENMEY & OHAJAI & POCYEL \\
\hline JABVIS & КАВYOB & LICHUA & MEXQIP & NENMIC & OHOJUQ & POJNIL \\
\hline JABVOY & KAFHEE & LICUPH & MIBPUI & NEQBAM & OHUCUP & POJPAF \\
\hline JAFGUS & KAMZED & LIHPAL & MIHCIP & NEVFEZ & OHUXUK & POMKAD \\
\hline JAGDUQ & KAMZIH & LIHPHM01 & MIHCUB & NEWFUQ & OKAYEE & POMLAE \\
\hline JAJHIL & KAMZON & LILDIT & MIHDOW & NEWGAX & OKUTIX & PONDAX \\
\hline JALTUL & KAPFAI & LILQAY & MIHDUC & NEWGEB & OKUTOD & PORFEH \\
\hline JAMWAV & KARMUL & LIMFES & MINXOW & NEXCOI & OLAJUG & POSYIF \\
\hline JAVQAY & KASHER & LIPHPR & MIQCIY & NEXQIQ & OLEXUY & POTCOQ \\
\hline JAVQEC & KAYFIZ & LIPKIE & MITHAY & NEYVES & OLEYUZ & POTFOT \\
\hline JAVVAD & KEGDUV & LIPTHF & MITHOM & NIGYAD & OLEZAG & POWZOQ \\
\hline JAYDOC & KEGHUZ & LITXER & MITJAA & NIMQIJ & OLEZEK & POYBUA \\
\hline JEDJEH & KELGOX & LIZKEK & MIZTEU & NIPDAR & OLIBUG & POYFOY \\
\hline JEFTAP & KEMNAR & LIZLEL & MIZTIY & NITLAD & OLICAN & PUHSEQ \\
\hline JEPSAY & KEQBAJ & LIZLOV & MOBQUP & NITLEH & OLICER & PUJTAP \\
\hline JEPSEC & KEQBIR & LIZLUB & MOBRAW & NIVTOB & OLIPAA & PUKJEK \\
\hline JEVSAE & KERHAQ & LOJGOG & MOBREA & NIVVET & OLIQUV & PUMGEJ \\
\hline JEVSEI & KEVNII & LOLNIJ & MOMCAS & NIWRAM & OLIVUA & PUPDIN \\
\hline JIBCOM & KEVQOR & LOLPEH & MOMCEW & NOGLUQ & OLIXOW & PURDAH \\
\hline JIBCUS & KEXDIA & LOQJAC & MONZOE & NOMJOO & OLUGOR & PUYFAQ \\
\hline JIBDAZ & KEZQAH & LOQJEG & MOQWOE & NOMJUU & OLUGUX & QACZAV \\
\hline JILRAX & KIBTEU & LOSMAH & MOQWUK & NONQUC & OMELOH & QACZEZ \\
\hline JIRQUW & KIQCAO & LOSMEL & MOQXAR & NOVQIY & OMITEJ & QACZID \\
\hline JIRTUZ & KIYSIU & LOVLAJ & MOSHOR & NOVWAW & OMITIN & QAGHAI \\
\hline JIRVAH & KIZPUE & LUBDAN & MOTYAV & NOXLAN & PACDAZ & QAGHEM \\
\hline JIRWIQ & КОВНEO & LUBQII & MOTYEZ & NOXLIV & PACLOU & QAGNOC \\
\hline JISDAQ & КОССЕК & LUBQOO & MOTYID & NUCZIU & PAFMEO & QAJGIR \\
\hline
\end{tabular}




\begin{tabular}{|c|c|c|c|c|c|c|}
\hline QAJNOE & QUPREY & RILNOP & SOLXUM & TOHLAD & VABFOU & WAJRAA \\
\hline QAKPEX & QUPRIC & RILNUV & SONJAG & TOKMEL & VACHIR & WAKGEU \\
\hline QAQZAJ & QUQWAA & RISPOY & SORYON & TOPQUK & VADKIU & WALQEF \\
\hline QARCOB & RABCIH & RISPUE & SOYKUM & TOPSOG & VAFHIT & WAMWAI \\
\hline QASKUQ & RACGUX & RIVCOO & SOZJUM & TOPSUM & VAFWEE & WAQLUV \\
\hline QATFIA & RACSAP & RIVDIJ & STLIEN & TOQVEA & VAGHIU & WARHIG \\
\hline QAYBIB & RAHLUH & RIYVUQ & SUHWEX & TOTXEF & VAGHOA & WARLOQ \\
\hline QAZRAK & RAJLET & RIYWAX & SUKWAW & TOTXIJ & VAGHUG & WATPEM \\
\hline QAZRIS & RAJLIX & RIZHEN & SULLIU & TOVQEA & VAJVIL & WAWNUD \\
\hline QAZROY & RAJLOD & RIZHIR & SUNROI & TOWXUY & VALYEM & WAWPAL \\
\hline QEBMIT & RAJLUJ & RIZHUD & SUNRUO & TOXMUO & VAMKOK & WAXZEA \\
\hline QEBMOZ & RAJMAQ & ROHLIJ & SUPLAQ & TOZQEE & VAPLUU & WAXZOK \\
\hline QEBMUF & RAKSAX & ROJGOM & SUPTAY & TOZQII & VAPMAB & WEBMAR \\
\hline QЕСТОH & RAKSEB & ROJGUS & SUPXOQ & TOZVAF & VAPYOB & WEBMOF \\
\hline QECTUN & RAKSIF & ROLFUT & SUQBAH & TUDBUP & VAQDIB & WECXAD \\
\hline QECVAV & RAKYAD & ROMMEL & SUQCOW & TUDHEF & VAQGAV & WEFJEW \\
\hline QELFIW & RANNAV & RORLOZ & SUQCUC & TUFMIQ & VASFEB & WEGMEA \\
\hline QESQUA & RANNEZ & RORMEQ & SUQRIF & TUJNER & VASPAH & WEKPIL \\
\hline QESZUJ & RANPIF & RUFFON & SUSDEP & TUMPAS & VATLAD & WEKYAM \\
\hline QEXYIB & RAPNAX & RUJWIC & SUSFAN & TURSEE & VAVKAE & WEQRIT \\
\hline QEYRUH & RAPNEB & RUKRAQ & TABGOS & TUVBER & VAZSUK & WEWXOL \\
\hline QIDPAU & RAPRAB & RUNLUH & TAFDAG & TUVROR & VAZTAR & WIBKEX \\
\hline QIFYOT & RAPREF & RUQRAW & TAFDEK & TUVSAE & VEBFEN & WIBNEA \\
\hline QINKAZ & RAQYUD & RUQYEH & TAFHEO & TUWSAF & VEGCUF & WIDWOV \\
\hline QINTIQ & RAWYET & RUQYIL & TAFSEZ & UBEVEC & VEGWEJ & WIHFAU \\
\hline QIQRUD & RAWYIX & RURLAR & TAGMEU & UBEWAZ & VEHVAF & WIJQIP \\
\hline QIQSAK & RAXLOR & RUVDOB & TAWWOD & UBEWON & VEMREK & WIKWUI \\
\hline QIQTUF & RAYFEC & RUYBUI & TAXVAP & UBIQOL & VEQYUL & WIMDIF \\
\hline QODTOS & RAZPOX & RUYCAP & TAZTAP & UBIQUR & VEQZAS & WISXIF \\
\hline QODTUY & RECXUS & RUYCET & TEBJEP & UBITEE & VESQUF & WIZGER \\
\hline QOHHOK & REDNOD & RUYCOD & TEGNEY & UBOHAU & VESREQ & WIZMEX \\
\hline QOKCEY & REFCUA & RUYYOZ & TEQPEK & UBOHEY & VESTES & WOCPIN \\
\hline QOKCIC & REHBOV & RUYZAM & TETNEL & UBOHUO & VEWXIE & WODMIL \\
\hline QOKCOI & REHBUB & SABKOV & TEXJUB & UBOSOT & VEWXOK & WOJBAY \\
\hline QOKCUO & REHSIG & SABKUB & TEZYOM & UCIYEK & VIKDAU & WOTQOL \\
\hline QOKDEZ & REHSOM & SAGCUY & TEZZAZ & UCIYOU & VINDOL & WOZTOU \\
\hline QOKDID & REKGOD & SAGDAF & TEZZIH & UCIYUA & VIXCUA & WUBBOK \\
\hline QOKFUR & REKGUJ & SAKROL & TIBCIQ & UCUNEL & VIXDUB & WUBSUH \\
\hline QOPQIV & REKHAQ & SAMNEZ & TIBCUC & UDEFIS & VIXRUP & WUCTUJ \\
\hline QOPRES & REMHEW & SEMHEX & TIBDAJ & UDEHOA & VOGFOM & WUCVAR \\
\hline QOPRIW & REMHIA & SENJUQ & TIBHER & UDEHUG & VOLDUV & WUCVEV \\
\hline QOQXID & REMHOG & SEQBUL & TIBTED & UDIZUC & VOPQOG & WUCZID \\
\hline QOSFIN & REPBUJ & SEQTEN & TIBTON & UFARIC & VOQBAE & WUDJEK \\
\hline QOSFOT & REPHID & SEQTIR & TIBTUT & UFUXAU & VOTLUL & WUJPOG \\
\hline QOXLOE & REPKUS & SETNAG & TIBVAB & UFUZUQ & VOWWEJ & WUKPOH \\
\hline QOYZOT & RETBEX & SEVBEA & TIBVEF & UHETEG & VUCKUZ & WULPOI \\
\hline QUDJEE & REVGII & SIGHAR & TIGDOC & UHUPIW & VUCLAG & WUQRAB \\
\hline QUFXEU & REWKIN & SIJZOA & TIGFAQ & UJENIG & VUDZID & WUQREF \\
\hline QUJGOR & REWPOY & SIMRIP & TIGMIF & UJIVOY & VULKOC & WUQTAD \\
\hline QUJHOS & REZBAZ & SIMRUB & TIGMOL & UJIVUE & VULPUN & WUSTUZ \\
\hline QUJHUY & REZBED & SIMZIX & TIHKEA & UJIWAL & VULSOK & WUSVAH \\
\hline QUKCEE & REZTUL & SIYXAZ & TINQUC & UJOPOY & VUMCAH & WUSXOX \\
\hline QUMDIL & REZVAT & SIZTUQ & TIPBID & UKODED & VUMCEL & WUWJAZ \\
\hline QUMHAH & RIBJER & SOCYIS & TISMIR & ULETIO & VUMDAI & WUWPAF \\
\hline QUNZII & RIBJIV & SOGXAN & TIZCEK & ULOPEQ & VUNGUG & WUXWER \\
\hline QUNZUU & RICCIP & SOJKEH & TIZXOP & ULUWUT & WABRIB & XABZUW \\
\hline QUPBIM & RIKTOU & SOKSEQ & TOCRIM & ULUXAA & WADHUF & XACBIN \\
\hline QUPRAU & RILLIH & SOLLUA & TOCROS & UMEPEH & WADKES & XАCBOT \\
\hline
\end{tabular}




\begin{tabular}{|c|c|c|c|c|c|c|}
\hline XACJER & YANLEE & ZEGNII & AJIZEY & BIYYEN & DALSUE & ESUJUX \\
\hline XACKUH & YANREK & ZEGNOO & AKOTAV & BIYYIR01 & DALWAO & ESUKEI \\
\hline XACROI & YAPXUI & ZEHNIJ & ALOWON & BOBDUR & DASMOZ & EWIWUC \\
\hline XAJPAZ & YAPYAP & ZENCEA & AMEVIX & BOBMOU10 & DEKYAT & FACLAW \\
\hline XARVER & YARPEM & ZEPKIO & AMPICC01 & BODXAT & DETLET & FAGCIZ \\
\hline XASWUJ & YASYUM & ZEPKOU & ANMESM & BOTKUQ & DETPIB & FAGVEP \\
\hline XASXAQ & YEBWAD & ZESGIN & APUJAW & BOXKOO & DICCIB & FAGWIU \\
\hline XASXEU & YEBWEH & ZESHAG & APUJEA & BOYRIQ & DIDCEY & FAHROV \\
\hline XASYEV & YEDKAT & ZEYSOL & APUJIE & BPOCNA & DIQKOD & FAJGEC \\
\hline XASYIZ & YELDAU & ZEZRAX & APXBZA & BUKBAK & DIQKUJ & FANSES \\
\hline XASYOF & YEMLAD & ZEZSOM & AQOVOR & BURDAT & DISTEE & FAQCEF \\
\hline XASYUL & YEMZUL & ZIBSEI & AQUNAB & BUXWUM & DIWWIP & FARKIS \\
\hline XASZEW & YEPZOI & ZIGWAN & AREYUR & BZCNOP & DNPNIS & FEBJUR \\
\hline XASZIA & YESVEX & ZIHCEY & AROTOQ & BZCSUR & DOFQOE & FECREK \\
\hline XAVFAB & YESWOI & ZIHCIC & ASEMOA & CABDOZ & DOFQUK & FEFJIJ \\
\hline XAWGOR & YETGIN & ZIHZAR & ASIVAZ & CABDUF & DOFWUQ & FEKLEM \\
\hline XECBUC & YEVSUN & ZIKDEC & ATAHAE & CADBOZ & DOJTOL & FEMNUG \\
\hline XECCAJ & YEVTAU & ZIMFIK & ATITOM & CADCAM & DOKHEQ & FERSAS10 \\
\hline XEDQEC & YEVTIC & ZISDAG & AVIFUG & CAFKEA & DOLJUJ & FERSCT10 \\
\hline XEFROP & YEWHAJ & ZITBEJ & AVONAA & CAHDUL & DOLNAT & FEXBAL10 \\
\hline ХЕКМАВ & YIDJAW & ZITBOT & AWOLAZ & CAMBIC & DOWVOA & FEYYEN \\
\hline XELSIQ & YIDTEK & ZIWZEK & BABKOE & CAMBOI & DPBNAC & FEZCAO \\
\hline XELSOW & YIDXAK & ZIYZOW & BABKUK & CASTAR & DPBNCN & FEZTEJ \\
\hline XENYAQ & YIFJEC & ZOGHOT & BACGUH & CATETH & DUCZOQ & FIGQER \\
\hline XENYEU & YIGSAI & ZOHVIB & BADPUS & CATHAG & DUFYOS & FINHOZ \\
\hline XENYIY & YIHBIA & ZOHXUP & BAFKAV & CAWTOJ & DUFZOT01 & FINHUF \\
\hline XETJEL & YIJFIG & ZOJKOY & BAGTUY & CAXXAA & DUFZUZ01 & FITSOQ \\
\hline XETJIP & YILZUO & ZOJKUE & BAHFOG & CAYCOU & DUNSUA & FIWQOR \\
\hline XEWFEK & YIMTOD & ZOJLIT & BAHFUM & CAZZEI & DUYJEM & FOLWIM \\
\hline XIBWIO & YIQMAM & ZOMKER & BAHGAT & CECATI & EBUQAT & FOLWOS \\
\hline XIBWOU & YIQMEQ & ZONJIV & BAKMUW & CENPEQ & EBUQEX & FOQBES \\
\hline XICNEC & YIQMIU & ZORBEN & BAMJEF & CENPOA & EBUQIB & FOQBUI \\
\hline XICNIG & YIQPIX & ZOTNUR & BAMNEJ & CEPFUY & EBUQOH & FOQWAJ \\
\hline XIDGIA & YIRRIA & ZOZPOT & BAMYOE & CEPKUD & EBUZOQ & FOQXOY \\
\hline XIGHAW & YISPUL & ZUBWOI & BAPHUV & CEQDIL & ECOGUY & FORPEH \\
\hline XIGHEA & YIXYIN & ZUFCIM & BAPJAD & CESLER & EDUNUM & FOVLUX \\
\hline XIQLUE & YIXYOT & ZUGJIU & BAQVEU & CESRAT & EFIWIZ & FTPNTF10 \\
\hline XITHEN & YOBKUV & ZUJWOR & BARCAZ & CIDLUW & EFURUS & FUFJUL \\
\hline XIVQAU & YOJKIR & ZUTJED & BASTEV & CINTOI & EGIMAI & FUFKIA \\
\hline XIVREZ & YOMCEI & ZUXDOL & BATCUU & CIPMIX & EGIMEM & FUMKUT \\
\hline XIXMOG & YOXLIG & ZUYGIJ & BAWHIQ & CIRFAK & EGIMIQ & FUNBEV \\
\hline XIXMUM & YOXWEN & ZUZCAY & BAXREX & CIRGEP & EGUDIT & FUNBIZ \\
\hline XIXNAT & YOZJUS & ZUZROB & BEBCAM & CISLIZ & EGUDOZ & FUNBOF \\
\hline XODKOQ & YOZKAZ & & BEBFIY & CIWXEL & EHGLFE10 & GABFEU \\
\hline XOFGAA & YUBVIA & $\mathbf{N a}$ & BECVUA & COCRIV & EHOSOJ & GABHAS \\
\hline XOFNOV & YUBVOG & ABAYUX & BEGWIU & COKROJ & EJOBOU & GAHDUO \\
\hline XONQOG & YUBVUM & ABAZEI & BEGWOA & COKZUX & EKERIV & GAHLOQ \\
\hline XOVYEM & YUVCEX & ABEHUK & BEHJEE & COLLEU & ELEQER & GANZOK \\
\hline XUCCON & YUVCOH & ABEPOM & BELBID & COSALS & ELUSEJ & GAWCIQ \\
\hline XUCRES & ZABFIR & ABXALA & BEMNIQ & COSJOJ & EMSBHC10 & GAWXAD \\
\hline XUNQEC & ZACFIS & $\mathrm{ABXBPC}$ & BENGEG & CULNIG10 & EMUVOX & GEDKAB \\
\hline XUPPUT & ZADSOM & ACFENA & BENJOU & CULNOM10 & ENOBUE & GEVKOH \\
\hline XUWHOM & ZAPKEG & ADINAC & BEZYIO & CUNKOL & EPNIST & GEZXIS \\
\hline XUWHUS & ZARLAF & AGONOZ & BICHUQ & CUQFUP & EQITED & GIJMOB \\
\hline XUYSIT & ZAWMIT & AGONUF & BICHUQ10 & CUQVAL & EROCUJ & GILJIU \\
\hline YADNOG & ZAWMOZ & AHAKEZ & BIGTAM & CURBOG & ESACUS & GIMYUW \\
\hline YAFYAF & ZAXFAF & AHALEA & BIYVOU & CUXROC & ESAZON & GIWLED \\
\hline YAHKEX & ZAXFEJ & AHALIE & BIYXUD & DALSEO & ESCTSB & GIWLIH \\
\hline
\end{tabular}




\begin{tabular}{|c|c|c|c|c|c|c|}
\hline GIYWAM & ILICEL & KATDEO & MAWREH & NATHQU & OLEMEX & QELZIQ \\
\hline GIZJII & ILICIP & KATGIV & MAYDAR & NAVSAE & OLEMIB & QEMKAU \\
\hline GOSBAR & ILOKID & KEHBUU & MAZBSN & NAWDIY & OLEWIL & QIHVUY \\
\hline GOVNAG & IMAVUN & KEKWAY & MAZQAF & NEJBUZ & OLIDIW & QIMQEI \\
\hline GOXWEV & INAROE & KEMFAJ & MAZQEJ & NEKRUQ & OLIXEM & QIMQIM \\
\hline GUKREJ & INARUK & KEQFOB & MDANAC11 & NELYAE & OLULIQ & QIWTEV \\
\hline GULQEJ & INASAR & KEQFUH & MDPCOR & NEMNAL & PABYIB & QOBKOH \\
\hline GUNRUC & IPOYAN & KERHIY & MEFLOY & NEPWEK & PAFQOC & QOGHUP \\
\hline GURQEP01 & IRABIM & KIBRES & MEMTUT & NEWYUJ & PALTEB & QOLVES \\
\hline GUTLUC & ISARID & KIBROC & MENFOA & NEXHON & PALYAC & QOLVIW \\
\hline GUTMAJ & ISORAJ & KICSIY & MEQVAF & NIMHAS & PALYEG & QOPGUX \\
\hline GUWVEZ & ITAJAO & KIGGAI & MEQXOV & NIMHEW & PAPJIZ & QOPQAN \\
\hline HADYOA & ITEROO & KIGGEM & MEWFOJ & NIQJAY & PATFOF & QOTRAS \\
\hline HAHNUZ & IVIDEW & KIMXOT & MEYZUL & NIQWAL & PAZTUF & QOTROG \\
\hline HANLOX & IVUCOR & KIWQUC & MIRMOP & NIRXAN & PBEGAN & QOTSAT \\
\hline HAPXIF & IWIVOZ & KOBHUE & MIWQAK & NISBAC & PCFENA & QOXKUJ \\
\hline HAPXOL & IWOJOT & KOCMUK & MIZBEC & NITMOS & PDACCO & QOZJIY \\
\hline HDHBNA & IWOXOH & KOCNAR & MOKMAA & NIZCUU & PEBFEH & QQQDHD01 \\
\hline HEDXIX & JAFPUC & KODBEK & MOLGEZ & NIZXUP & PEHMAQ & QQQFJD02 \\
\hline HEDXOD & JAHJIL & KOFCIR & MOSNIR & NODVAD & PEHPIB & QQQFMA01 \\
\hline HEDXUJ & JANMAM & KOJGIZ & MOTLEM & NOQROA & PEJROL & QUFSUF \\
\hline HEFSIU01 & JAPKOA & KOVLOW & MOTLIQ & NOVGOU & PEKNEY & QUFTAM \\
\hline HEHJEJ & JARCOU & KUBKAT & MPIXPS & NOWVOK & PELCEO & QUGKOS \\
\hline HETCIS & JAXROP & KUBKEX & MUBGUL & NOXLOB & PESJEC & QUPLAO \\
\hline HEWXOW & JAZKIE & KUBKIB & MUDQOR & NOYBAE & PESTEM & QUPLES \\
\hline HFCATH & JEGFIK & KUCJEX & MUDQUX & NPENRS & PEZTET & QUQFIR \\
\hline HFCATH10 & JEHHUZ & KUDJOI & MUDRAE & NQXSNK & PIDCIO & RACRES \\
\hline HIDCOM & JELMIW & KUTGIP & MUMNIR & NTBZOV & PIGBUC & RACRUI \\
\hline HIDLAH & JEPWAC & LABKOO & MXPENA & NUDSIO & PIPGEA & RADXID \\
\hline HIGGAF & JEVPUV & LAFDOL10 & NAABZH & NUDSOU & PIRMOS & RAKYEH \\
\hline HIJVAX & JILLOF & LAGJOS & NABCRA & NUGBAS & PKJNAC & RASPAC \\
\hline HIKLIW & JISHEY & LAJHIN & NABCRB & NUGLAC & PKOJSI & RASPEG \\
\hline HIKLOC & JISHIC & LAKWAV & NABPEX & NUPRUL & POGDAQ & RATNIJ \\
\hline HIZMIM & JODDIP & LALLEP & NABSUF & NUVBIP & POGZAM & RATNOP \\
\hline HOKZAI & JOFJUJ & LASVUW & NAHPHT & NUYFAO & POLREN & RATNUV \\
\hline HORSUC & JOGDOY & LAZRUZ & NAHPTB10 & OBUMIH & PONPEN & REDBAD \\
\hline HOSXAO & JOJZAJ10 & LAZSAG & NALJUF & OBUMON & PORQIW & REFHAL01 \\
\hline HOSXES & JOLWIQ & LAZSEK & NALKAM & OBUMUT & POWZUW & REFHAL02 \\
\hline HOTGIG & JUBGUI & LEXDIB & NALKEQ & OBUNAA & PPYMSI & REJBAJ \\
\hline HOWGOP & JUBGUI01 & LEXWAM & NAMOTS & OCACAW & PTHAZN & REJBEN \\
\hline HOZVEX & JUBYOU & LEYTAK & NAMXAC & OCEWAU & PUDWIU & REJRED \\
\hline HUCJEU & JUGSIN & LIBCUU & NANAPH & OFOWUB & PUGKEH & REMGUL \\
\hline HUDJEV & JUKQEL & LIFPIZ & NANBAF & OFOXAI & QACYEY & REVWAQ \\
\hline HUFRAB & JUKQIP & LIRFIB & NANBEJ & OFUNIM & QADYOK & REVWEU \\
\hline HUKYOB & JUPQOA & LOFNAV & NANBIN & OFUNOS & QAFWAW & REZPAN \\
\hline HUPZOH & JUQPAM & LOJFUL & NANBOT & OGEBEH & QAHGUB & RILBAP01 \\
\hline HUTZEB & JUQPEQ & LOLYAM & NANBUZ & OGIROL & QAJCUZ & RILLED \\
\hline HUXBUX & JUVBOR & LONBUL & NANMOE & OGIRUR & QAJDEK & RILMAA \\
\hline IBUTUU & JUXRUP & LOXVEZ & NANMSO & OGOHOH & QAJNAQ & RILMEE \\
\hline ICAGIC & JUXTAX & LOYVIE & NANNAR & OHBZPA & QALMEV & RILMII \\
\hline IDEGED & JUYSEB & LUCIDS & NAPHBZ & OHBZPB & QASKAW & RIPRUD \\
\hline IFONUM & KACWIV & LUVHIT & NAPHCU & OHBZPC & QASZUF & RIXTEX \\
\hline IGUBIV & KAFPIR & LUZLAT & NAPHSX & OKATOJ & QAWSEM & RODYEO \\
\hline IKEZED & KAFYUM & LUZLEX & NAPOCA & OKOYOC & QAWSIQ & ROMXIA \\
\hline IKILOD & KAMHEL & MAMMOC & NASALN & OLAXEE & QAYLUX & ROMXOG \\
\hline IKILUJ & KAMHIP & MAMMUI & NASDIU & OLAXII & QAZREO & ROMXUM \\
\hline IKULEF & KANNES & MAPKAP & NASOXH & OLEKUL & QAZRUE & ROQFEI \\
\hline IKUSOW & KARFIS & MAWRAD & NASUAM & OLELAS & QAZSAL & ROQFIM \\
\hline
\end{tabular}




\begin{tabular}{|c|c|c|c|c|c|c|}
\hline ROQFOS & SUKMEQ & UDAPUK & VUSWIP & XEDBIR & YUGDOT & ALIKOV \\
\hline RUJDIJ & SUKQEU & UDOTOW & VUSZAK & XETVAT & YUHJAM & ALIKUB \\
\hline RUJGEI & SULCIL & UDOVAK & VUZXOD & XETVEX & YUKXOR & APHOUB \\
\hline RULNIV & SULJAK & UDUJIM & WABMET & XEXJUF & YUXZUM & AQEPUH \\
\hline RURYOS & SULZEE & UFATAW & WADKAO & XEYDAG & ZACRAW & AQEQAO \\
\hline RUVDUH & SUNBEI & UFIMUR & WAHFAM & XIFBAP & ZAFYIO & AQOVIL \\
\hline RUVFET & SUNRIC & UGUNOZ & WAJJAS & XIFBET & ZAGSIJ & AREZAY \\
\hline RUVMAW & SURGIV & UHARUQ & WAJJEW & XIHPUZ & ZAGYIP & ATITEC \\
\hline RUYQOR & SUSSOO & UHUPOC & WANZEQ & XILFIH & ZALKUS & AVAVOI \\
\hline RUZRAF & SUVXAI & UJEGOF & WAPPAE & XIPTAR & ZALXEP & AVETUQ \\
\hline SACKEM & TABRAQ & UKAWAE & WARNEI & XISWEB & ZAPCOI & AVEVEC \\
\hline SAFJEO & TABROE & UKAWEI & WARQAH & XIVVAZ & ZAPCOI10 & AVEVIG \\
\hline SAFJIS & TADLUF & UKOPOZ & WASWOC & XIVVED & ZAVGAE & AWEZOR \\
\hline SAJJES & TADNER & UKOPUF & WAWFOP & XIVVIH & ZAXGEK & BACTOO10 \\
\hline SALCNA & TAFLUI & UKOQAM & WAXPAM & XIVVON & ZAYYED & BACTUU10 \\
\hline SALFIU & TAJSUS & ULEVAI & WEGFOD & XIVVUT & ZEBSUU & BACVAC 10 \\
\hline SALGCO & TALBOY & UMITOZ & WEGGEU & XIVWAA & ZEMSAL & BADNIE \\
\hline SAMNOJ & TALDAL & VABQAQ & WESHIL & XODRUD & ZEPJUZ & BADPOM \\
\hline SANMAL & TAPVOV & VAFGEO & WEZFOW & XOHSIW & ZEXCAG & BANCOI \\
\hline SAVSAJ & TARDOF & VAHCAJ & WIBNOK & XOTXIN & ZIGQUA & BANCUO \\
\hline SAXYLF & TASCEV & VAKDAM & WIGTEL & XOVMUQ & ZILVAR & BAPGAA \\
\hline SBHOCD & TAVNEJ & VAKDIU & WILGUT & XOVYUC & ZILVEV & BAQKOT \\
\hline SBZDZS & TAXTIV & VALYUD & WIMCIE & XURBIV & ZILVIZ & BARCIH \\
\hline SCATUR & TECCIN & VAMNEC & WINJAE & YACDUB & ZILVOF & BARMAI \\
\hline SEBJUE & TEJTOR & VAMSIL & WIPXAU & YAFCEN & ZILVUL & BASVIB \\
\hline SEHHAO & TEKFAQ & VARJOO & WIPXEY & YAHKIB & ZILWEW & BASWAU \\
\hline SEKCIU10 & TEXKAI & VARZIX & WIVNEU & YAJZOY & ZIMLOW & BATWEY \\
\hline SELTUY & TIBDUD & VATBIC & WIWXEF & YAMBOD & ZIMMIR & BAXSUO \\
\hline SENBIW & TIBVIJ & VAXJIN & WIWXOP & YANRIO & ZODRAL & BAXYII \\
\hline SESXOD & TIGGEV & VEPFEB10 & WIWYAC & YAPPEK & ZOGHOS & BDTASH \\
\hline SESXUJ & TIHJOJ & VEQTEQ01 & WIWYEG & YAXSUL & ZOHYAW & BEBFAP \\
\hline SIBZIM & TIHKAW & VEQYAR & WOHSIV & YAYWUQ & ZOHYIE & BECDAP \\
\hline SIBZOS & TIRZEZ & VEQYEV & WOKTIZ & YAZLEQ & ZOPPOJ & BEDBER \\
\hline SIGLEZ & TITWIC & VEQYIZ & WOKTOF & YAZQAR & ZOPWUW & BEGBIZ \\
\hline SIPHEE & TIVTIB & VESTIW & WONNUI & YENKUX & ZOPXOR & BEGWEP \\
\hline SIRHEG & TIZKES & VESTOC & WOPJOA & YENQUD & ZOQPOK & BEHLOQ \\
\hline SIRHIK & TOCYAL & VIHNUV & WOTVOQ & YERFIK & ZOTPAZ & BEHLUW \\
\hline SIWHIP & TODHEZ & VIHQIM & WOTVUW & YEXBUY & ZOWMED & BIDYES \\
\hline SMERQS01 & TOFMOQ & VIHROT & WOTWAD & YEZBUA & ZOZHOL & BIFMIM \\
\hline SMSTCO & TOGWIV & VIKGUR & WOTWOR & YEZCAH & ZUBJUB & BIFZAR \\
\hline SNITYR10 & TONYAW & VIWHUE & WOZTUA & YIDXAK & ZUBKAI & BINZAZ \\
\hline SNQXSO & TOZPED & VIWQEX & WUCVEV & YIJWUJ & ZUQMED & BIQNAQ \\
\hline SNTOSP & TPMNAE & VIXDAH & WUZNIO & YIJXEU & ZUQVUC & BIQNEU \\
\hline SOCCIW & TUBDID & VIXDEL & XABBOS & YIJXIY & ZURVAJ & BIQNIY \\
\hline SOCCOC & TUBMUY & VIYDUC & XACRUO & YIWYAE & ZZZAXD10 & BITWEG \\
\hline SOCSOS & TUBNAF & VODXUH & XACSID & YOFMAH & ZZZPOW01 & BIXLAV \\
\hline SOHKIJ & TUBNOT & VOHXOF & XAFNUN & YOHLUC & ZZZUPI01 & BIZJOJ \\
\hline SOJCOJ & TUDCAW & VOKNEO & XAFPAV & YOLXIG & & BOBWIY \\
\hline SOJKIL & TUHMUE & VOKPUG & XAMWIR & YOPGOZ & $\mathbf{K}$ & BOBWOE \\
\hline SOJLIM & TUJMUG & VOKRAO & XAMWOX & YOPNUM & ACEHAR & BOFRET \\
\hline SOMCEC & TUJNIV & VOLJAH01 & XAMWUD & YOWGAS & ACITOV & BOXVEP \\
\hline SOPXAW & TUKDAE & VOPKAM & XАMXAK & YOYCEU & ADAREC & BPANSK \\
\hline SPHENC & TULLUH & VORTOL & XAQGOL & YOYROT & AFITIS & BPOPKI \\
\hline SPHIND & TUQWAD & VOTCAI & XAVHUX & YOYSAG & AFITOY & BPTGLK \\
\hline SSULAN & TUQWOR & VOYYAJ & XAVXOH & YOYXIT & AFITUE & BUTGAY \\
\hline SUDLAE & TUTGIY & VUHHUB & XAZMUG & YOZJOM & AGUDUB & BUWVUK \\
\hline SUDMEJ & UBIHAO & VUKGUD & XEBVIJ & YUBMUD & AGUFAJ & BZPENK01 \\
\hline SUDWAP & UDAPOE & VUNHAN & XEDBEN & YUCKUC & AKIKIO & CABFAN \\
\hline
\end{tabular}




\begin{tabular}{|c|c|c|c|c|c|c|}
\hline CADJUM & EDOQAP & GENCEH & ICOMIW & KAMBZH & KTNASO10 & MOTMAJ \\
\hline CAFVAH & EGOREX & GEPRUO & IFASOX & KATBOW & КTPHEB & MOTMEN \\
\hline CAGFOG & EJEBAW & GEPSAV & IFASUD & KATBUC & КTPHEB01 & MOTMIR \\
\hline CALYOD & ELECIH & GEPTUQ & IFATAK & KATCAJ & KTYSUH10 & MPTCRH \\
\hline CALYUJ & ELEHOS & GERXOQ10 & IFATEO & KATCIR & KUCJIB & MUDQAD \\
\hline CASHUZ & ELEJAG & GEYDAP & IFONOG & KBZILT & KULWOD & MUDQIL \\
\hline CASLUD & ELEQIV & GIFJUA & IGOJOD & KCATCR & KUPFIK & MUMNOX \\
\hline CATDAC & EMEZIF & GINMEV & IGONOH & KCATFE & KUVTAW & NABLUX \\
\hline CAZHEQ & EMIZEF & GIPBOW & IKULIJ & KCROFE & KUVTEA & NAGTEV01 \\
\hline CECNUT & EMUVUD & GITCIV & IKUSIQ & KEBPEM & LACHEC & NAKVEA \\
\hline CEJMEJ & EMUYOA & GITYAJ & IMIHER & KEKLUH & LACKEF & NATJUN \\
\hline CELLIO & EMUYUG & GIWXIT & IPUQOZ & KEQZAH & LAJYOK & NAYXAM \\
\hline CELWIZ & EQITIH & GIXWEP & IPUSER & KEQZEL & LAJZAX & NAYXEQ \\
\hline CEMQEQ & EROBES & GIYLAB & ITAKAP & KEVTAG & LALJUD & NAZMUW \\
\hline CEYLEX & EROJEA & GOBDUW & IVADEO & KHBSLF & LAPQUO & NBTPCU \\
\hline CIGVAP & ETAJEO & GOBFEI & IWOKUA & KHCBZO10 & LAQXOQ & NCRKTC10 \\
\hline CIRDUC & ETUCOL & GOBFOS & JAKXEY & KHDBEZ & LAQXUW & NEJCEK \\
\hline CMXPIC10 & EVAREY & GOBFUY & JAKXOI & KHDIAN & LAWFIY & NEJCIO \\
\hline COHRIA & EVAVOM & GOGBIN & JARFIR & KHDPAC & LAWLAW & NELKIY \\
\hline COTMED & EVAWUT & GOGBUZ & JARFOX & KHDPAC12 & LEFVEX & NERWIQ \\
\hline COVPEI & EWOJOP & GOGHEP & JARFUD & KHHPAL & LEHRAR & NERWOW \\
\hline COWSAI & FAFPAE & GOKWEI & JAYQAB & KHNDPA11 & LEPXIN & NEVGAW \\
\hline COWSEM & FAFPEI & GOKYUA & JEFZOJ & KHNIBO & LEQVAE & NEZDUR \\
\hline COYDEZ & FAGSIP & GOKZEL & JETKEY & KHPHAL & LESRIK & NIBJUD \\
\hline CUDWUT & FAJFUR & GOTHOM & JICGUX & KHQUDQ & LEWNEG & NICJIS \\
\hline CUDXAA & FAKDUQ & GOXWIZ & JIVKII & KHQUIN & LEXZUJ & NICXEC \\
\hline CULJOI10 & FANWAS & GOYGEG & JIVXER & KIBDOT10 & LEYBAS & NIFSAW \\
\hline CUPPEI & FAPNAL & GUJXAK & JIVXIV & KICKAI & LEYBEW & NIMRUW \\
\hline CUSFUR & FETJOD & GUTMEN & JIVXOB & KIFCOR & LEYZIY & NIMSEH \\
\hline CUXMEN & FETJUJ & GUWVID & JOFGEQ & KINACP & LIDVID & NIYNEO \\
\hline DAMMEJ & FEXBEP & GUZCEJ & JOMDUK & KINMUP & LIHYAC & NODPAX \\
\hline DATBAB & FEXTEH & GUZDIO & JOMFAS & KIVKOP & LIKCOX & NOFTOR \\
\hline DAXNAR & FICNIO & HACFOH & JOMFEW & KIVZOE & LINXAH & NOKKAZ \\
\hline DAYQUP & FICYUL & HAGTOY & JOMFIA & KIXWET & LOCXAC & NOMKEF \\
\hline DECDUK & FIDMEK & HANSAQ & JOMFOG & KIXYAR & LOJFOF & NOQREQ \\
\hline DEJWUK & FIPFUF & HARHOX & JOMFUM & KIXYEV & LOLXUF & NOQRIU \\
\hline DEKMEL & FIPGAM & HARSAU & JOQNAE & KIXYIZ & LOLYEQ & NOSKUB \\
\hline DEKSUH & FIRQOM & HARSEY & JOSLOS & KIXYIZ01 & LOSHAC & NPHINA \\
\hline DEXSOO & FIWTOU & HASTEA & JOVCIG & KIXYOF & LOTWOG & NPXPKT10 \\
\hline DIBYUI & FIWVUC & НВХСТК & JOVVAR & KIXZUM & LUGZUI & NPXUNK \\
\hline DIBZAP & FIYDOG & HECSAJ & JOYJOW & KIYBEZ & LUHBAR & NQOXSK \\
\hline DIBZET & FIYDUM & HEFLAF & JOYJUC & KIYZIB & LUHXER & NQXSNK \\
\hline DIFMUA & FLMENK & HEFPAJ & JUCLAU & KIZVOE & LUHXOB & NUDROT \\
\hline DIMXIG10 & FOKHIW & HEQZUY & JUCLUO & KMEPBZ & LUTWAY & NUZZEN \\
\hline DOCCIH & FUCPEY & HERCIQ & JUKZOE & KMERQU & MAVSUX & OBOLAS \\
\hline DOLKTC & FUDXIL & HIGTAS & JULCAU & KMOOCT10 & MAXFUM & OBOLEW \\
\hline DORGOG & GADNAB & HIJFOV & JULHIH & KNPCYM10 & MEBHIK & OBOQUR \\
\hline DUCXAA & GAHLIK & HIJFUB & JULQEM & KNPHEH & MEDGIL & OBUJEA \\
\hline DUCZEG & GAHNOS & HILTEB & JUPQAM & KNPHEH01 & MEDGOR & OCABEZ \\
\hline DUFBIP & GAHNUY & HITHEX & JUTVAV & KNQXSO & MEQXEL & OCIRUN \\
\hline DUGBAI01 & GAHPAG & HIZLUX & JUTVEZ & KPBUCO & MERCUH & OCXCPK \\
\hline DUGBOW01 & GAKVIX & HIZMEI & JUTVID & KPHPOP10 & MIGWUU & ОСХСРК01 \\
\hline DUJROP & GANSIX & HOODKI01 & JUTVOJ & КРНTHT & MIMNIF & ODAJEI \\
\hline DULGIA & GANZIE & HORRAH & JUTVUP & KPHTNB10 & MIZDAA & ODUDAS \\
\hline DUNSOU & GARWUR & HORREL & JUTWAW & KPNACN & MOGLOJ & OGEBIL \\
\hline EBENEE & GASVEB & HORRIP & JUXPOH & KTFPHT & MOLJOM & OGUKAC \\
\hline EBPCDC01 & GEBCUL & HORROV & KAFJEG & КТFРНT01 & MOMDIB & OGUKEG \\
\hline EDODOQ & GEJNOY & HOSVOA & KAKNUF & KTMPNM & MOPHAA & OGUKIK \\
\hline
\end{tabular}




\begin{tabular}{|c|c|c|c|c|c|c|}
\hline OGUROX & PXMPYK & SAFQAR & UCARAR & WELNUW & XIZLUN & ZUBMAK \\
\hline OHOJIE & QACGEH & SAHTAW & UDUKUZ & WETROC & XIZMIC & ZUFHIR \\
\hline OJOPIM & QAGSIB & SAKXOR & UFINAY & WEXQAR & XOBWAM & ZUYYIB \\
\hline OKATUP & QAHHAI & SAMFUH & UHOFIG & WEXQEV & XODSEO & ZZZAVY01 \\
\hline OKAVAX & QATHAU & SAMGAO & UHOFOM & WIDMUR & XODSIS & ZZZAVY10 \\
\hline OLEKIZ & QAYHED & SAXRAK & UJABUC & WIMCAW & XOFNAH & ZZZHDA01 \\
\hline OLEKOF & QAZTOA & SAXTEQ & UKORIV & WIPSET & XOTMIC & ZZZOVA01 \\
\hline OLEWOR & QAZTUG & SEBQEV & UKUCIM & WIPSIX & XOVZAJ & \\
\hline OLEXEI & QAZVAO & SEFJIW & UKUCOS & WIQFUX & XUBYUO & Mg \\
\hline OLEXIM & QAZVES & SENHUO & UKUCUY & WIRFUY & XUJSOK & AJAXOY \\
\hline OLEZOU & QELKIB & SEYBUT & UKUDAF & WISXAX & XUKLAQ & AKIDED \\
\hline OLINUS & QELZAI & SEYBUT10 & UKUDIN & WIVREY & XUMTII & ALEBIC \\
\hline OLIYAJ & QEQTAH & SIPSUF & UKUDOT & WIYDUD & XUNDEP & ALEBOI \\
\hline OLIYAJ01 & QESNIL & SIQXIZ & UKUDUZ & WIZJIY & XUQDAO & ALOHAK \\
\hline OPENBO10 & QIHXIO & SIYPIZ & UKUFAH & WOFHOO & XUXFEB & ALOHEO \\
\hline OPHENB10 & QIRKIL & SOCQUW & UKUFEL & WOFHUU & XUZDOL & ALOHOY \\
\hline OXTETK & QIVWEX & SOCSIM & UKUFIP & WOFJAC & YACVAZ & ANAPHS \\
\hline PAGYUR & QIVWIB & SOCSIM10 & UKUFOV & WOFJEG & YACVIH & AQFVMG \\
\hline PAJGUC & QIWTIZ & SOGGAW & ULEFIA & WOGTAN & YAMYUG & ASUBAR \\
\hline PAJHEN & QIZXAY & SOGGAW10 & ULETEK & WOHSOB & YANDOG & ATONIG \\
\hline PAPDOZ & QOBXUA & SOVSIF10 & ULETOU & WOKXAV & YANGOJ & BAFKAV \\
\hline PATDOD & QOGJAX & SUCCUO & ULEVEM & WOPCIN & YARMUZ & BAWZII \\
\hline PATDUJ & QOKDUP & SUDPIQ & ULOWAT & WOTWUX & YARMUZ10 & BAYYOQ \\
\hline PAVXAL & QOKFEB & SUGXEX & UMADER & WOXTEI & YEBDIS & BEHBAS \\
\hline PAXRAH & QORWUP & SUVPOO & VABCOR & WOYCES & YEFFAQ & BEHBEW \\
\hline PAXSIQ & QOXKOD & TABRUK & VADWED & WOYCIW & YEMLEH & BEXDEN \\
\hline PAYQEL & QOZYEJ & TAGKIW & VAKKIB & WOYCOC & YEMZOF & BIXBEP \\
\hline PAYSAJ & QOZYOT & TAGKOC & VAMSOR & WOZVAI & YENMIN & BOFSAQ \\
\hline PAYSOX & QUBXUG & TAGMOE & VENDAT10 & WUCSAO & YESSAQ & BOYFAW \\
\hline PBZPOS10 & QUCTOX & TAMMAV & VEPFAX10 & WUHBOQ & YEWYEE & BZMGIC \\
\hline PDNMET & QUQWAA & TCTOSN & VEQTIU01 & WUHDUY & YEZFOY & CAMNEK \\
\hline PEBBIH & QURMUL & TEJTIL & VEQWET & WUHFAG & YIKDEB & CAWTID \\
\hline PEBROD & QURNAS & TEZJAJ & VICCOZ & WUHVOK & YIYFOB & CEPTUM \\
\hline PEBRUJ & QUSBEL & TEZJEN & VIHMII & WUWSEM & YOLZAA & CEPVOI \\
\hline PEBSAQ & RABLIQ & TIFDAN & VIHQAE & XACSAV & YOWNAZ & CIPVAY \\
\hline PEDVAV & RAFSUM & AN01 & VIHQOS & XANTKI10 & YOWNED & COGBUV \\
\hline PEDVEZ & RAGHOW & TIJCUK & VIY & XEBVAB & YUBMEN & CULXAI \\
\hline PINYEQ & RAHBAD & TIJRAF & VOFTUF & XEBVEF & YUHJUG & CULXEM \\
\hline PMESUL & RAKYIL & TIQQOZ & VOGREO & XEDCIS & YUHTAW & CULXIQ \\
\hline PNIOCO & RASCIX & TIWPIY & VOHXUL & XEDCOY & YUKDAJ & DENCUU \\
\hline PNOMEK & REMGEV & TMALNK & VOTCEM & XEKNIK & YULBEM & DIHFEF \\
\hline POGLOM & REMGIZ & TOLCEC & VUYZEU & XEMBEW & YUMRON & DIHFIJ \\
\hline POHGUO & REPBOD & TOPDIL & VUZWUI & XEMJUU & YUPJUO & DIHQAM \\
\hline PONYOG & RERJED & TOPDUX & VUZXIX & XЕМКАВ & YUVYIX & DITFAN \\
\hline POPICR & REZPER & TOVKEU & WABDOT & XEMWUH & YUVYOD & DOCFOQ \\
\hline POPICR01 & RIBHUF & TOXNOJ & WACRUO & XESKEL & ZAKMAZ10 & DOQLUQ \\
\hline POPICR11 & RIMBOE & TOZNUR & WAGJAP & XESSOD & ZAPQOW & DPMGEN \\
\hline POPICR12 & RIRMUA & TOZPAZ & WAJGET & XESWEX & ZAWREU & DUJKUO \\
\hline POPICR13 & RISREQ & TOZPIH & WAPNAC & XETTUL & ZAYGIP & DUYFOS \\
\hline POQLUC & RIZMAO & TUBNEJ & WAPNAC10 & XEYDEK & ZEFNIH & ECESAG \\
\hline POQNOY & RIZZOP & TUCCOJ & WAPTEM & XEYVOM & ZEKVIU & ECESIO \\
\hline POQNUE & ROJVOB & TUDCEA & WAQTEN & XIBCOA & ZEWHUE & ECESOU \\
\hline POTTUN & ROSDUY & TUFDAZ & WAVCUR01 & XICNOM & ZIFRAH & ECESUA \\
\hline PTRPHT & RUFSIU & TUKCUX & WAVCUR02 & XIFVEN & ZITSUQ & EFOKAL \\
\hline PULRUJ & RUHMOW & UBIRAY & WAYTOF & XIVCOU & ZITSUQ01 & EFOKEP \\
\hline PULSAQ & RUNSAU & UBITII & WECXIL & XIVCUA & ZIWZIO & EGFEMG10 \\
\hline PUNTAT & SAFNES & UBITOO & WEHKEZ & XIVSOK & ZIXWOS & EGIJUZ \\
\hline PUYGIZ & SAFNOC & UBUZIA & WEKBET & XIYGIV & ZOXDAR & EMEZA \\
\hline
\end{tabular}




\begin{tabular}{|c|c|c|c|c|c|c|}
\hline EMIFAH & IBEZUK & MOSJAF & QUJSOD & VAXJAF & AVEYUV & JAKBOM \\
\hline ENATIW & IBIBEA & MUYRUT & QUJTEU & VIBXIN & AVEZAC & JASFUE \\
\hline ENATOC & INDYMG & NAFAMG10 & QUJTIY & VIBXOT & BABGAM & JEHJEL \\
\hline ERUPAI & INUMOT & NEBZOJ & RADXEZ & VIYPIC & BCRCIH10 & JEMZAC \\
\hline ESCUHM & INUMUZ & NECBEC & RAJZOR & VIYPUO & BOQPEC & JOQLAC \\
\hline ESONUV & IROMOR & NELCAI & RAKYOR & VIYRAW & BOWHAW & JOSCOJ \\
\hline ESOPAD & ISAREZ & NOHJOJ & RARCIW & VOKSAP & BZCAIM10 & JUSQIX \\
\hline ESOPEH & ISUBUT & NOHJUP & RARCUI & VOPCEI & CAHAPY & KAQSAW \\
\hline ESOPIL & IWOYEY & NOXVIF & RASHEY & WABHAJ & CAHPAL10 & KOLZEQ \\
\hline FAHSAI & JAFZUL & NUDXAL & RATRIN & WADLOD & CAMANX & LAXZUF \\
\hline FAYMIB & JAGBAU & NUDXEP & REJFAN & WECXOR & CAMELL10 & LAYBAO \\
\hline FEDQAG & JAPFOV & NUZXAH & REJWEI & WECXUX & CANAPO & LICHAG \\
\hline FEDQUA & JAPFUB & ODAKEJ & RIBYIK & WEGHIZ & CAPCGL10 & LOSPOY \\
\hline FEGQAJ & JASDUC & OFOZUE & RIZJAL & WEHMUR & CAPHTH & LUBLUP \\
\hline FIQGUH & JIRFAR & OFUBAS & RIZJUF & WEHNAY & САРНTH01 & LUMCEB \\
\hline FOJWOQ & JIVSAI & OFUCAT & ROLGAA & WEHNEC & CASALA01 & MILKUN \\
\hline FOMGAP & JIWDEY & OFUCIB & RUGNEM & WEHNIG & CATPAL & MODZEK \\
\hline FOMPIG & JONKAY & OGIZAF & RUGTES & WEVJAI & CEVGUF & MOTFOQ \\
\hline FOMPOM & JUQTUK & OGUKOQ & SAMKAS & WIDYIR & CINFIO & MOTFUW \\
\hline FOXBID & KAFGII & OLIBOA & SEBJUE & WIKXAP & COLJES & MUCWOW \\
\hline FSALCM & KAFHOP & OMARID & SIDNAU & WUHYAZ & CUZPES & MUCWUC \\
\hline GAGLIJ & KASTUT & PALGUE & SINDAU & WURZIS & DOQMAX & MUJFEC \\
\hline GANQAN & KEGNIT & PAPBUD & SIWMOA & WURZOY & EDAMIF & MUJTOA \\
\hline GANQER & KEHBOO & PAPCEO & SIWMUG & WURZUE & EDAREG & NICSUN \\
\hline GANQIV & KEQTUV & PAPTUV & SOBTAE & WUYKUW & EDUNIA & NORPUF \\
\hline GANQOB & KOKWIQ & PAXMEG & SOWWOQ & WUYLAD & EFOJIS & NUXHET \\
\hline GATLUI & KOLBIW & PEFXIH & SUKWAW & WUYLEH & EFOJOY & OJUZAU \\
\hline GAWMIA & KOLDAQ & PHMGBE & TACXEB & XARZEV & EFOJUE & OLEYAF \\
\hline GECZAP10 & KOLVIQ & PIHMUO & TACXIF & XAVKUA & EGENUZ & OLOQEL \\
\hline GEPBIM & KUVJAM & PIPCOG & TAFHAK & XEXLAN & ELOGIV & OMARAV \\
\hline GEPBOS & KUVJIU & PIPCUM & TAGKAO & XIMBAW & FACQUW & PESTOW \\
\hline GEPBUY & LEMJOC & PIPDAT & TAGKES & XITDUZ & FAHRUB & PIFWIK \\
\hline GETMUN & LEMJUI & POFNON & TAHKUJ & XIYRAY & FEDQEK & PPDCAE \\
\hline GEXMEB & LEMKAP & POHGIC & TASBIY & XUDSEU & FEDQIO & PRSMCA \\
\hline GIKCOS & LETZOZ & PPOSMG & TASBOE & XUHTUP & FEDSIQ & QAFNAM \\
\hline GIXXEQ & LEXDAT & PUBREJ & TASBUK & YASKEI & FEDWIU & QEMKAU \\
\hline GOHRUQ & LIBVUN & PUFGEC & TATNAD & YESVIB & FEFNUZ & QEMKEY \\
\hline GOHSAX & LIBWEY & PUNRIZ & TAVZUL & YIQGOU & FEKQER & QESGEA \\
\hline GOLPIG & LIBWIC & PXMGIM & TAWHEE & YIQGUA & FIBVAN & QIVGIL \\
\hline GUBSIF & LIDKEO & PXMGPY & TAYSIV & YIQHAH & FUFFIV & QODHIA \\
\hline GUQTAN & LISRUA & QAGLUG & TIBNIB & YIQHEL & FUVCII & QOPPEQ \\
\hline GUTBOM & LOHBIT & QAGMAN & TOCYOZ & YIZGIX & GADFOG & QOPPIU \\
\hline GUZYUV & LOHBOZ & QAGMER & TUFCEC & YUGGUC & GASDAF & RAKYUX \\
\hline GUZZAC & LUSJUE & QAGMIV & TUXYOA & YUGHAJ & GAWKUK & RATKEC \\
\hline GUZZIK & LUWLOE & QAQSUW & UDAQIZ & YUHJIU & GECYES & RELKAU \\
\hline HAGVOA & LUWMAR & QAYPOV & UFANIY & ZARMEK & GEZYOZ & REMNAY \\
\hline HALZOJ & LUWMEV & QEBCEF & UFIZIS & ZEYKIX & GUHNEC & REVGOO \\
\hline HAMBEC & MAKHUB & QESJAZ & UGABAF & ZIQLAM & GUTGUX & REZZIF \\
\hline HAMFAC & MAKJAJ & QESKAA & UMEHEZ & & GUYBEH & REZZOL \\
\hline HAMHOS & MEFNOA & QESKII & UNATEI & $\mathbf{C a}$ & HACBET & RIJMAY \\
\hline HAYTIK & MFPDMG & QIBCEJ & VACSIB & AFOSAP & HASQAT & ROGRAG \\
\hline HEDSUE & MGABES & QIBCIN & VAQBEV & AFOSUJ & HATPIB & RUFMUA \\
\hline HETZUB & MGAPYC10 & QIBCOT & VAQBOF & AFOVAS & HEWBUG & TAQGAT \\
\hline HIGQET & HBZA10 & QIBCUZ & VAQBUL & AKUPEB & HUJZUH & TAQHAU \\
\hline HIJWEC & MGNTSP & QIBDOU & VAQLEE & AROWOT & IDECOJ & TAQREI \\
\hline HIRXOV & MGPCPH & QIFWOR & VAQLII & ATADUU & IJIDIO & TEFBEL \\
\hline HUXCIM & MGSACA10 & QIFWUX & VASNUZ & ATAZOK & IJIDUA & TEGMAT \\
\hline HUXHAJ & MINKOJ & QOPPAM & VAXHUX & AVEYOP & IPUSOB & TEGMEX \\
\hline
\end{tabular}




TEPWIU
TEPWOA
TIBNEX
TODJEB
TORPEV
TOWVOQ
TOWVUW
UHEKUN
UMISOY
UMISUE
UNEMIJ
VAQBIZ
VAQCAS
VAYGOR
VIJQEK
VIQBEC
VOPBUX
VUGZUS
VURHEV
WAZMEP
WEXLOA
WIKXET
WOTCOX
WOYBAN
XACVUS
XAVLAH
XAWFIK
XEQMOV
XMXCBP
XOBVUF
XORRIF
XUNBEN
YAZBOQ
YAZQEV
YINDAA
YINHIM
YOMJAL
ZAYCUX
ZEWSUP
ZEXCEK
ZOWKAX
ZOWKEB
ZUGTAW
ZUZSAO


Table S4: $\pi-\pi$ interaction energies (in $\mathrm{kcal} / \mathrm{mol}$ ) of the various benzene dimers in the presence of metal ions at MP2/6-31G* level of theory.

\begin{tabular}{cccccc}
\hline M & & S-M & PD-M & TA-M & TB-M \\
\hline & - & 0.14 & -0.07 & -1.58 & \\
& $\mathrm{Li}^{+}$ & -3.71 & -3.60 & -0.48 & -7.18 \\
$\mathrm{Na}^{+}$ & -2.88 & -3.08 & -0.47 & -6.06 \\
$\mathrm{~K}^{+}$ & -2.15 & -2.18 & -0.52 & -5.37 \\
$\mathrm{Mg}^{2+}$ & -11.36 & -13.06 & -3.31 & -15.08 \\
$\mathrm{Ca}^{2+}$ & -7.60 & -7.86 & -1.93 & -12.17 \\
\hline
\end{tabular}

Table S5: Interaction energies of metal ions with the benzene dimers at MP2/6-31G* level of theory.

\begin{tabular}{ccccccc}
\hline $\mathrm{M}$ & S-M & PD-M & TA-M & TB-M & SW1 & SW2 \\
\hline $\mathrm{Li}^{+}$ & -40.57 & -40.24 & -35.61 & -42.31 & -64.01 & -38.37 \\
$\mathrm{Na}^{+}$ & -26.95 & -26.93 & -22.81 & -27.43 & -43.64 & -23.93 \\
$\mathrm{~K}^{+}$ & -18.67 & -18.49 & -15.29 & -20.16 & -30.49 & -15.73 \\
$\mathrm{Mg}^{2+}$ & -123.43 & -124.92 & -113.65 & -125.43 & -189.69 & -135.02 \\
$\mathrm{Ca}^{2+}$ & -73.68 & -73.74 & -66.29 & -76.53 & -120.68 & -78.21 \\
\hline
\end{tabular}

Table S6: Geometries (in $\AA$ ) of the various metal- $\pi-\pi$ complexes of benzene dimers obtained at the MP2/6-31G* level of theory.

\begin{tabular}{|c|c|c|c|c|c|c|c|c|c|c|c|c|}
\hline \multirow{2}{*}{$\mathrm{M}$} & \multicolumn{2}{|c|}{ S-M } & \multicolumn{2}{|c|}{ TA-M } & \multicolumn{2}{|c|}{ TB-M } & \multicolumn{3}{|c|}{ PD-M } & \multirow[t]{2}{*}{ SW1 } & \multirow{2}{*}{$\begin{array}{c}\text { BM1 } \\
\mathrm{R}_{1}\end{array}$} & \multirow{2}{*}{$\frac{\text { BM2 }}{\mathrm{R}_{1}}$} \\
\hline & $\mathrm{R}_{1}$ & $\mathrm{R}_{2}$ & $\mathrm{R}_{1}$ & $\mathrm{R}_{2}$ & $\mathrm{R}_{1}$ & $\mathrm{R}_{2}$ & $\mathrm{R}_{1}$ & $\mathrm{R}_{2}$ & $\mathrm{R}_{3}$ & & & \\
\hline- & & 3.768 & & 4.868 & & 4.868 & & 3.387 & 1.653 & & & \\
\hline $\mathrm{Li}^{+}$ & 1.913 & 3.548 & 1.935 & 4.911 & 1.935 & 4.868 & 1.913 & 3.200 & 1.400 & 1.977 & 1.932 & 2958 \\
\hline $\mathrm{Na}^{+}$ & 2.378 & 3.577 & 2.400 & 4.885 & 2.400 & 4.868 & 2.378 & 3.300 & 1.400 & 2.403 & 2.400 & 3.330 \\
\hline $\mathrm{K}^{+}$ & 2.823 & 3.630 & 2.849 & 4.915 & 2.849 & 4.868 & 2.823 & 3.300 & 1.500 & 2.802 & 2.848 & 3.720 \\
\hline $\mathrm{Mg}^{2+}$ & 1.957 & 3.308 & 1.969 & 4.704 & 1.969 & 4.868 & 1.957 & 3.000 & 1.500 & 2.043 & 1.973 & 2.835 \\
\hline $\mathrm{Ca}^{2+}$ & 2.449 & 3.408 & 2.464 & 4.790 & 2.464 & 4.868 & 2.449 & 3.100 & 1.400 & 2.489 & 2.471 & 3.265 \\
\hline
\end{tabular}


Table S7: The metal ion affinities (in $\mathrm{kcal} / \mathrm{mol}$ ) of benzene monomer in the presence and absence of another benzene at the MP2/6-31G* and MP2/6-311++G** level of theory.

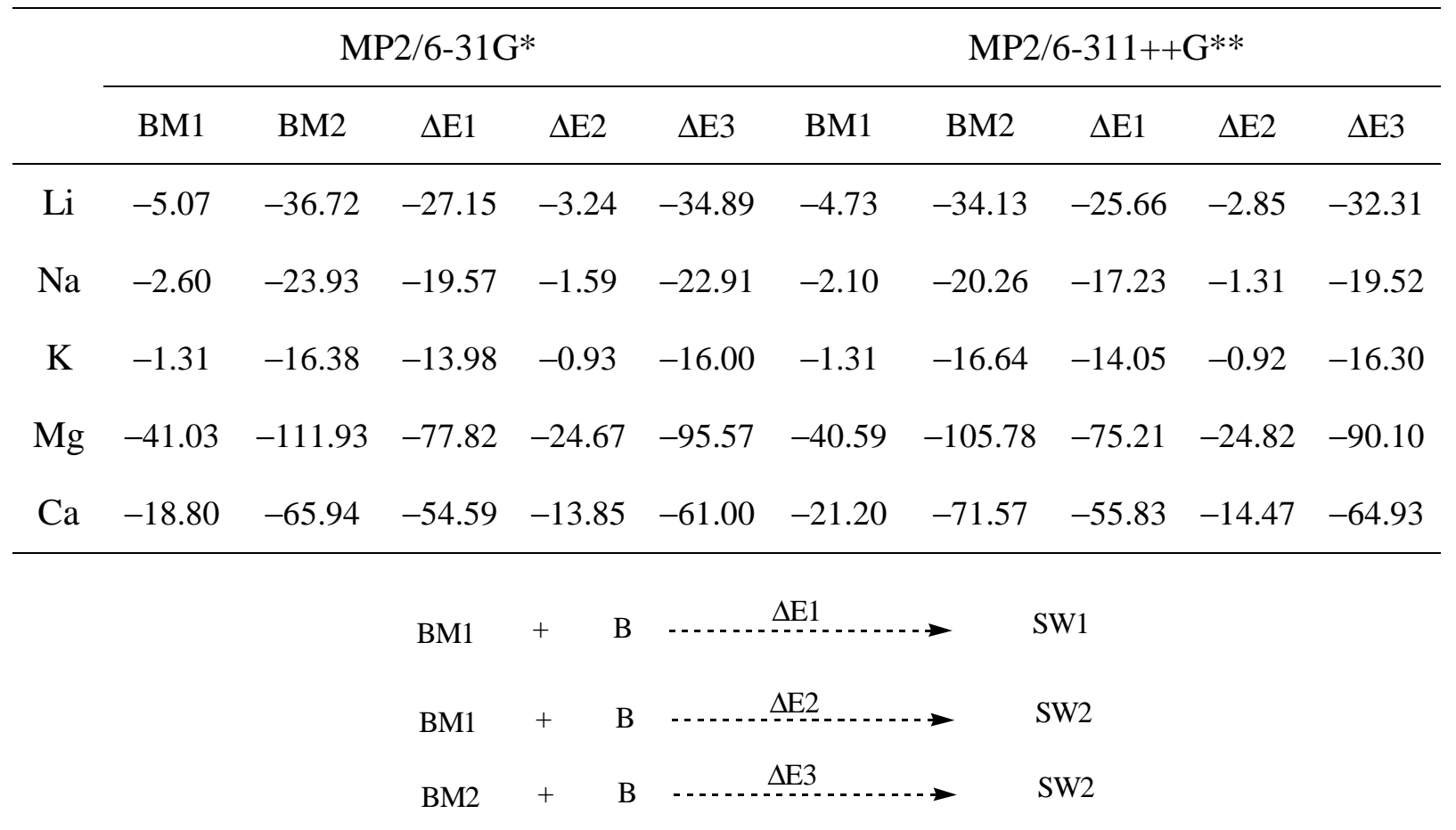

Table S8: Total energies (in hartrees) of M- $-\pi-\pi$ complexes at MP2/6-31G* level of theory.

\begin{tabular}{ccccccc}
\hline $\mathbf{M}$ & $\mathbf{S}-\mathbf{M}$ & PD-M & TA-M & TB-M & SW1 & SW2 \\
\hline $\mathrm{Li}^{+}$ & -470.230214 & -470.231775 & -470.222762 & -470.234595 & -470.278108 & -470.228389 \\
$\mathrm{Na}^{+}$ & -624.630172 & -624.631500 & -624.624462 & -624.634409 & -624.662813 & -624.625688 \\
$\mathrm{~K}^{+}$ & -1061.943191 & -1061.944572 & -1061.938996 & -1061.948028 & -1061.965912 & -1061.938704 \\
$\mathrm{Mg}^{2+}$ & -661.935678 & -661.948128 & -661.927738 & -661.946328 & -662.059699 & -661.962035 \\
$\mathrm{Ca}^{2+}$ & -1139.159585 & -1139.161644 & -1139.147985 & -1139.164714 & -1139.239530 & -1139.166262 \\
\hline
\end{tabular}


Table S9: Total energies (in hartrees) of $\mathrm{M}-\pi-\pi$ complexes at MP2/6-311++G** level of theory.

\begin{tabular}{ccccccc}
\hline $\mathrm{M}$ & $\mathbf{S - M}$ & PD-M & TA-M & TB-M & SW1 & SW2 \\
\hline $\mathrm{Li}^{+}$ & -470.479426 & -470.482327 & -470.471151 & -624.634409 & -470.421630 & -470.471099 \\
$\mathrm{Na}^{+}$ & -624.882613 & -624.885236 & -624.876148 & -161.664286 & -624.845525 & -624.872838 \\
$\mathrm{~K}^{+}$ & -1062.372046 & -1062.374540 & -1062.366560 & -1061.948028 & -1062.340949 & -1062.361394 \\
$\mathrm{Mg}^{2+}$ & -662.196700 & -662.202367 & -662.180419 & -661.946328 & -662.094345 & -662.209006 \\
$\mathrm{Ca}^{2+}$ & -1139.614591 & -1139.617805 & -1139.600317 & -1139.164714 & -1139.519230 & -1139.612492 \\
\hline
\end{tabular}

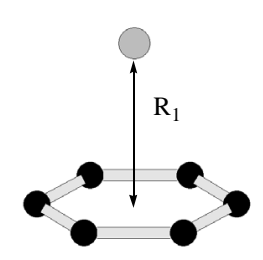

BM1

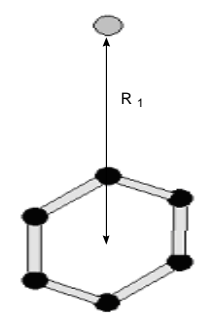

BM2

$\mathrm{Li}^{+}(-34.13) ; \mathrm{Na}^{+}(-20.26) ; \mathrm{K}^{+}(-16.64) ; \mathrm{Mg}^{2+}(-105.78)$; $\mathrm{Ca}^{2+}(-71.57)$

$\mathrm{Li}^{+}(-4.73) ; \mathrm{Na}^{+}(-2.10) ; \mathrm{K}^{+}(-1.31) ; \mathrm{Mg}^{2+}(-40.59) ; \mathrm{Ca}^{2+}$ $(-21.20)$

Scheme S1. The two cation- $\pi$ configurations considered in the study along with theirMP2/6311++ $\mathrm{G}^{* *}$ interaction energies (in $\mathrm{kcal} / \mathrm{mol}$ ). The metal ion approach is along the $\Pi$ face in BM1 while in BM2 is along the molecular plane. 


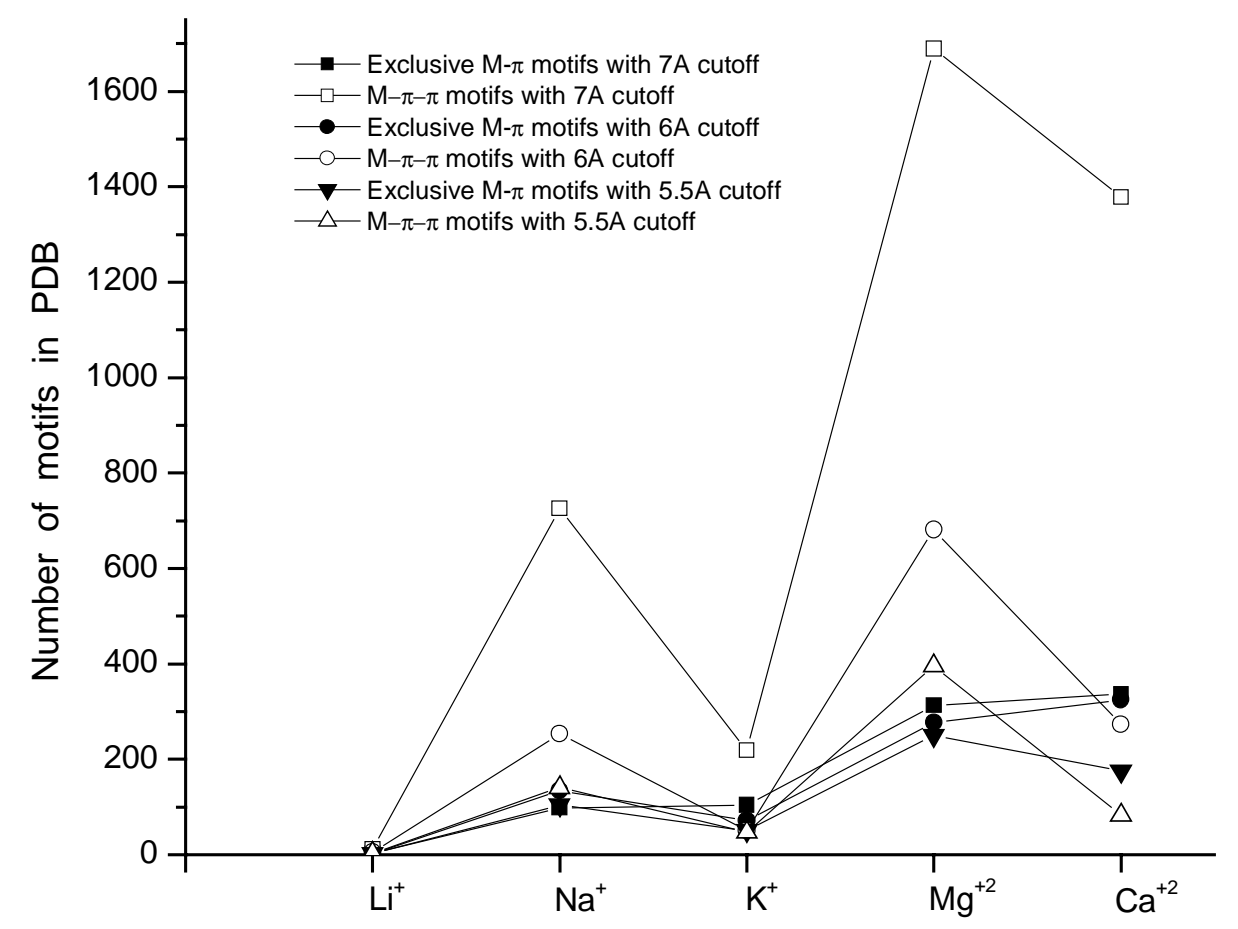

Figure S1: Statistics on the PDB with varying cutoff distances between the centroids of the aromatic rings and the metal ions. 


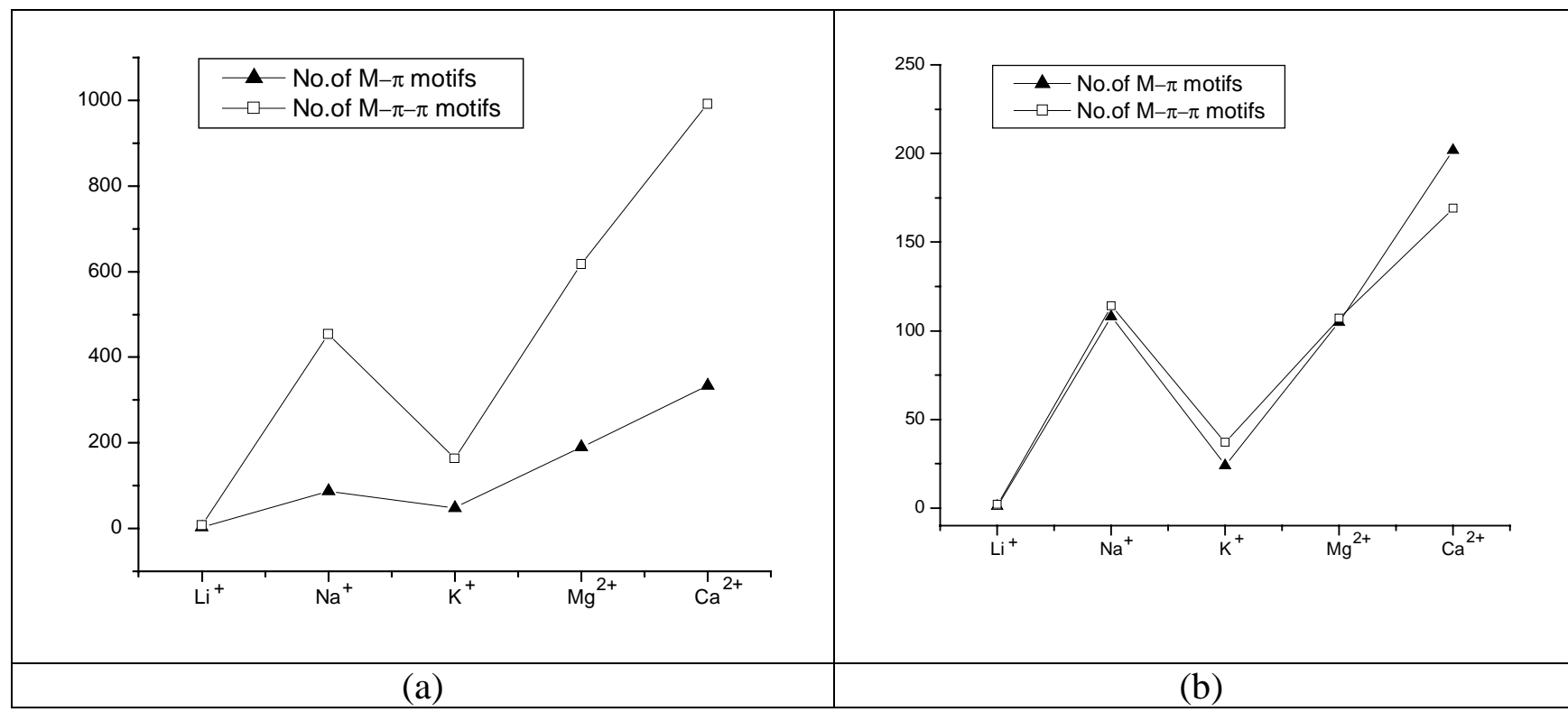

Figure S2: Statistics of $\mathrm{M}-\pi$ and $\mathrm{M}-\pi-\pi$ motifs in PDB considering the imidazole ring of the histidine moiety as the second $\pi$ but located at a distance larger than the cutoff values of $7 \AA$ (a) and $6 \AA$ (b). 

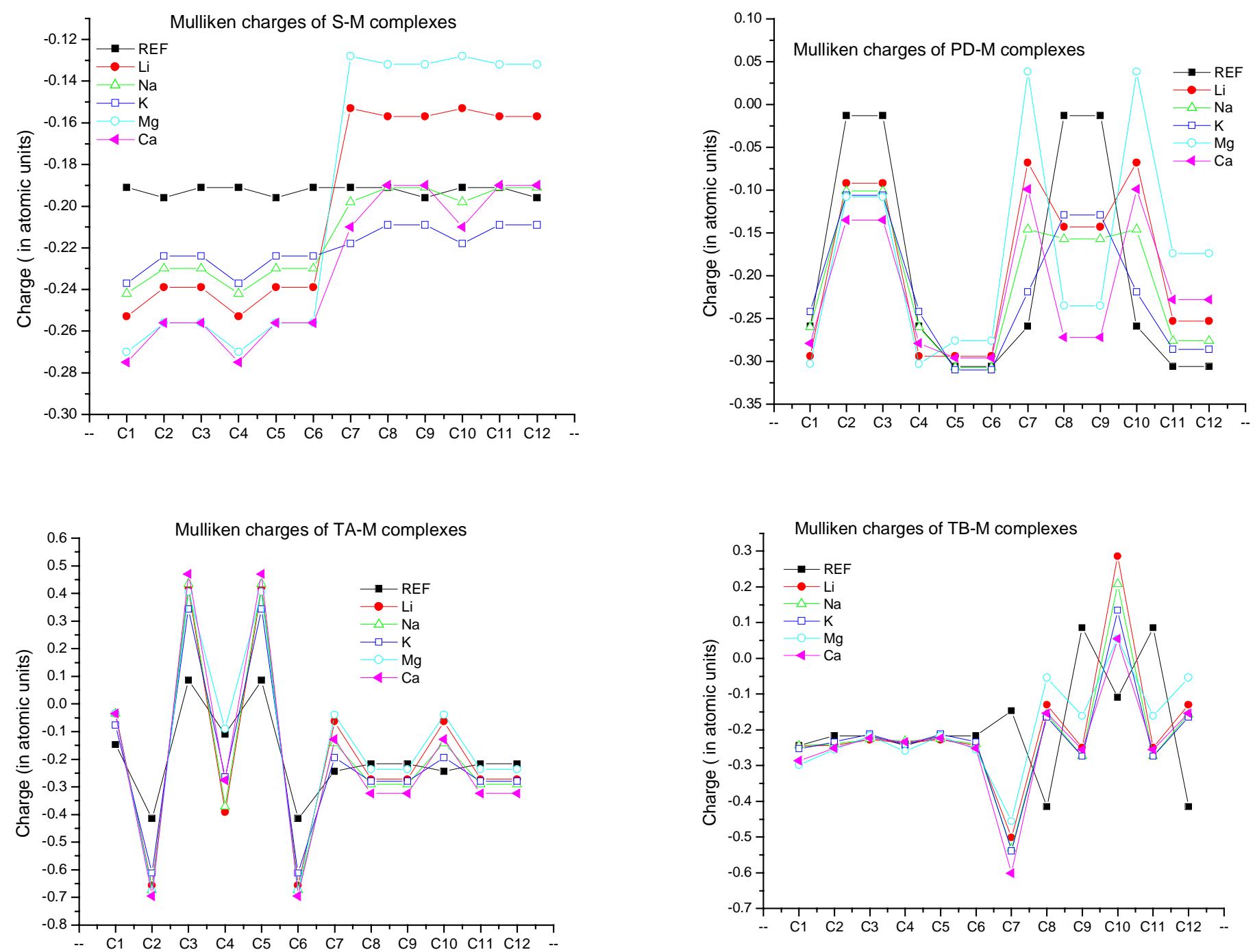

Figure S3: Mulliken charges of the carbon atoms in various cation $-\pi-\pi$ conformations considered. In all the complexes the carbon atoms $\mathrm{C} 1-\mathrm{C} 6$ correspond to the benzene ring far from the metal ion, where as the $\mathrm{C} 7-\mathrm{C} 12$ correspond to the nearer benzene ring to the metal ion. 

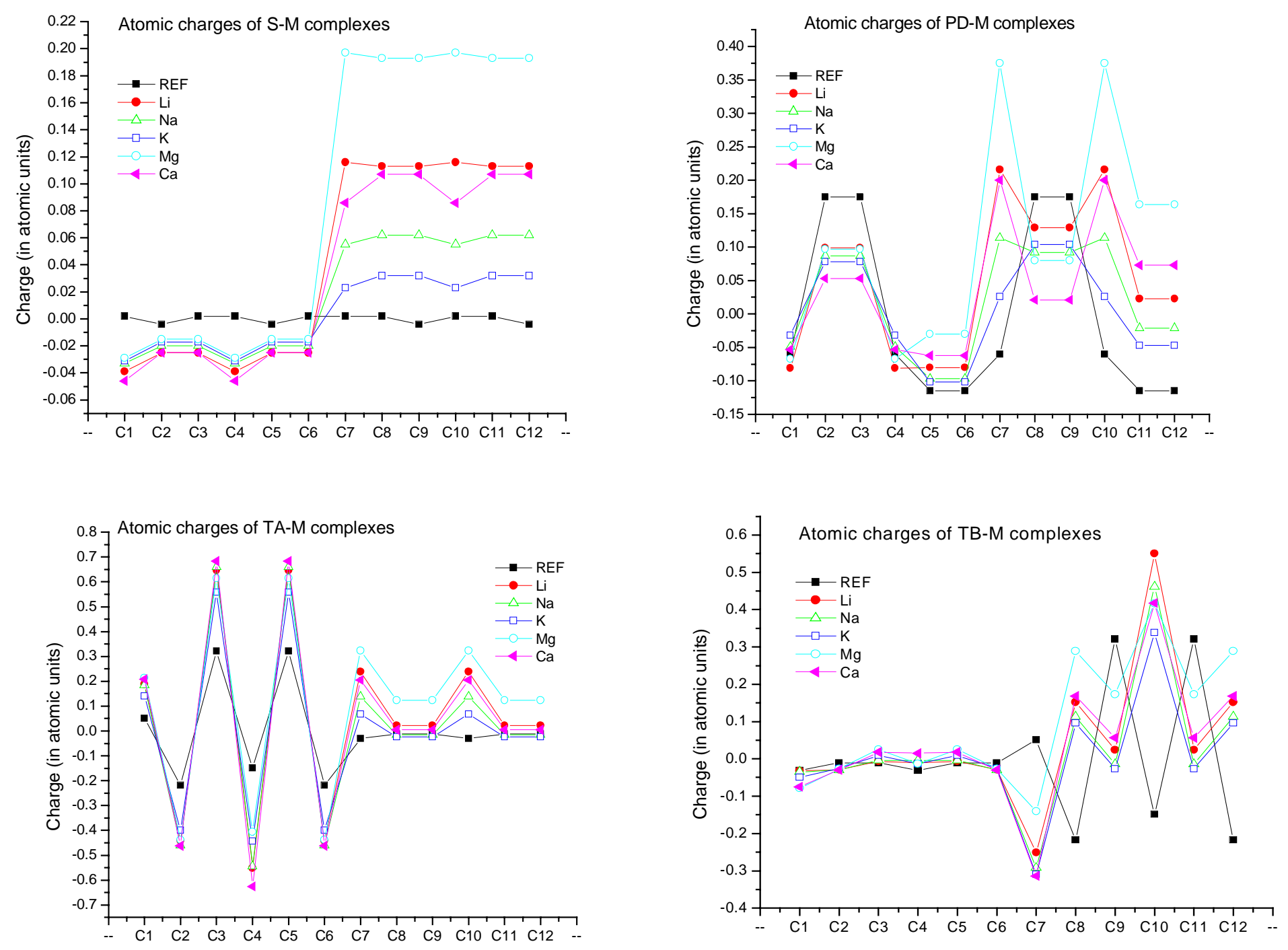

Figure S4: Total atomic charges of the carbon atoms in various cation $-\pi-\pi$ conformations considered. In all the complexes the carbon atoms C1-C6 correspond to the benzene ring far from the metal ion, where as the $\mathrm{C} 7-\mathrm{C} 12$ correspond to the nearer benzene ring to the metal ion. 